\title{
FUTURE STABILITY OF THE FLRW FLUID SOLUTIONS IN THE PRESENCE OF A POSITIVE COSMOLOGICAL CONSTANT
}

\author{
TODD A. OLIYNYK
}

\begin{abstract}
We introduce a new method for establishing the future non-linear stability of perturbations of FLRW solutions to the Einstein-Euler equations with a positive cosmological constant and a linear equation of state of the form $p=K \rho$. The method is based on a conformal transformation of the EinsteinEuler equations that compactifies the time domain and can handle the equation of state parameter values $0<K \leq 1 / 3$ in a uniform manner. It also determines the asymptotic behavior of the perturbed solutions in the far future.
\end{abstract}

\section{INTRODUCTION}

The Einstein-Euler equations for an isentropic perfect fluid with a linear equation of state and a positive cosmological constant are given by

$$
\begin{aligned}
& \tilde{G}^{i j}=\tilde{T}^{i j}-\Lambda \tilde{g}^{i j} \quad(\Lambda>0), \\
& \tilde{\nabla}_{i} \tilde{T}^{i j}=0,
\end{aligned}
$$

where $\tilde{G}^{i j}$ is the Einstein tensor of the metric

$$
\tilde{g}=\tilde{g}_{i j} d x^{i} d x^{j},
$$

$\Lambda$ is the cosmological constant, and

$$
\tilde{T}^{i j}=(\rho+p) \tilde{v}^{i} \tilde{v}^{j}+p \tilde{g}^{i j} \quad\left(\tilde{g}_{i j} \tilde{v}^{i} \tilde{v}^{j}=-1\right)
$$

is the perfect fluid stress energy tensors with the pressure determined by the equation of state

$$
p=K \rho \quad(K \geq 0) .
$$

On spacetimes of the form $\left[T_{0}, \infty\right) \times \mathbb{T}^{3}$, the Friedmann-Lemaitre-Robertson-Walker (FLRW) solutions to (1.1)-(1.2) represent a homogenous, fluid filled universe that is undergoing accelerated expansion. In [12],

the future non-linear stability of these solutions under the condition $0<K<1 / 3$ and the assumption of zero fluid vorticity was established by Rodnianski and Speck using techniques developed by Ringström in [11. Subsequently, it has been shown [7, 8, 13, that this future non-linear stability result remains true for fluids with non-zero vorticity and also for the equation of state parameter values $K=0$ and $K=1 / 3$, which correspond to dust and pure radiation, respectively. It is worth noting that the stability result for $K=1 / 3$ established in [8] relies on Friedrich's conformal method [5, 6], which is completely different from the techniques used in [7, 12, 13, for the parameter values $0 \leq K<1 / 3$.

In this article, we introduce a new method for establishing the future non-linear stability of FLRW solutions to (1.1)-(1.2). The advantage of our method is threefold: (i) it leads to a relatively compact stability proof, (ii) the parameter values $0<K \leq 1 / 3$ can be handled in a uniform manner, and (iii) it relies on a conformal transformation that compactifies the time domain to a finite interval, which we expect will be useful for numerical simulating solutions globally to the future. A further advantage of our method is that it can be easily adapted to handle the case $K=0$; we will present the details in a separate article.

Our approach to solving the Einstein-Euler equations (1.1)-(1.2) shares much in common with the methods employed in [11, 12, 13, in that it is based on a particular choice of wave gauge that brings the Einstein-Euler system into a form that is suitable for analyzing behaviour in the far future via energy estimates. We remark that, for us, a suitable form means a symmetric hyperbolic system of the type analyzed in Appendix B. The key difference between our approach and [11, 12, 13] is that we do not analyze the physical spacetime metric directly, but instead use a conformal metric as our primary gravitational variable. It is worth noting that our method does not rely on the conformal field equations of Friedrich [5, 6]. 
In order to describe our approach, we first must fix our notation. The spacetimes that we consider are of the form $M=(0,1] \times \mathbb{T}^{3}$. We use $\left(x^{I}\right),(I=1,2,3)$, to denote (spatial) periodic coordinates on $\mathbb{T}^{3}$, and $t=x^{0}$ to denote a time coordinate on the interval $(0,1]$ where the future lies in the direction of decreasing $t$. Unless stated otherwise, we use lower case Latin letters, i.e. $i, j, k$, to index spacetime coordinate indices that run from 0 to 3 while upper case Latin letters, i.e. $I, J, K$, will index spatial coordinate indices that run from 1 to 3 . Partial derivatives with respect to the coordinates $\left(x^{i}\right)$ will be denoted by $\partial_{i}=\partial / \partial x^{i}$. The fluid-four velocity $\tilde{v}^{i}$ is assumed to be future oriented, which is equivalent to the condition

$$
\tilde{v}^{0}<0 \text {. }
$$

As indicated above, we do not work with the spacetime metric (1.3) directly, but instead use the conformally transformed metric

$$
g_{i j}=e^{-2 \Phi} \tilde{g}_{i j}
$$

As has been demonstrated previously in [5, 6, a key technical advantage of the conformal approach is that it turns global existence problems into local ones via the compactification of spacetime. In our setting, this amounts to us being able to obtain solutions globally to the future by solving the conformal Einstein-Euler equations on a finite time interval. An additional benefit of the conformal approach is that it allows us to account for the leading order effects of accelerated expansion through a judicious choice of the conformal factor.

Under the conformal transformation (1.6), the Einstein equations (1.1) transform as

$$
G^{i j}=T^{i j}:=e^{4 \Phi} \tilde{T}^{i j}-e^{2 \Phi} \Lambda g^{i j}+2\left(\nabla^{i} \nabla^{j} \Phi-\nabla^{i} \Phi \nabla^{j} \Phi\right)-\left(2 \square \Phi+|\nabla \Phi|_{g}^{2}\right) g^{i j}
$$

or equivalently

$$
-2 R_{i j}=-4 \nabla_{i} \nabla_{j} \Phi+4 \nabla_{i} \Phi \nabla_{j} \Phi-2\left[\square \Phi+2|\nabla \Phi|^{2}+\left(\frac{1-K}{2} \rho+\Lambda\right) e^{2 \Phi}\right] g_{i j}-2 e^{2 \Phi}(1+K) \rho v_{i} v_{j}
$$

where we employ the notation $\square=g^{i j} \nabla_{i} \nabla_{j}$ and $|\nabla \Phi|^{2}=g^{i j} \nabla_{i} \Phi \nabla_{j} \Phi$ and we have introduced the conformal fluid four-velocity

$$
v^{i}=e^{\Phi} \tilde{v}^{i}
$$

We note that the four-velocity future orientation condition (1.5) is equivalent to

$$
v_{0}>0
$$

where $v_{0}=g_{0 j} v^{j}$. Unless otherwise specified, we raise and lower all coordinate tensor indices using the conformal metric $g_{i j}$ and its inverse $g^{i j}$ as appropriate.

Letting $\tilde{\Gamma}_{i j}^{k}$ and $\Gamma_{i j}^{k}$ denote the Christoffel symbols of the metrics $\tilde{g}_{i j}$ and $g_{i j}$, respectively, the difference $\tilde{\Gamma}_{i j}^{k}-\Gamma_{i j}^{k}$ is readily calculated to be

$$
\tilde{\Gamma}_{i j}^{k}-\Gamma_{i j}^{k}=g^{k l}\left(g_{i l} \nabla_{j} \Phi+g_{j l} \nabla_{i} \Phi-g_{i j} \nabla_{l} \Phi\right) .
$$

Using this, we can express the Euler equations (1.2) as

$$
\nabla_{i} \tilde{T}^{i j}=-6 \tilde{T}^{i j} \nabla_{i} \Phi+g_{l m} \tilde{T}^{l m} g^{i j} \nabla_{i} \Phi
$$

The wave gauge we employ is defined by the vanishing of the vector field

$$
Z^{k}=X^{k}+Y^{k}
$$

where

$$
\begin{gathered}
X^{k}:=g^{i j} \Gamma_{i j}^{k}=-\partial_{i} g^{k i}+\frac{1}{2} g^{k l} g_{i j} \partial_{l} g^{i j} \\
Y^{k}=-2 \nabla^{k} \Phi+\mu \delta_{0}^{k}
\end{gathered}
$$

and $\mu$ is to be specified.

For the conformal factor, we choose

while for $\mu$, we choose

$$
\Phi=-\ln (t)
$$

$$
\mu=\frac{2 \Lambda}{3 t}
$$

With these choices the vector field (1.9) becomes

$$
Z^{k}=X^{k}+\frac{2}{t}\left(g^{k 0}+\frac{\Lambda}{3} \delta_{0}^{k}\right)
$$


Remark 1.1. Since we want the conformal factor $e^{2 \Phi}$ to become unbounded towards the future to account for accelerated expansion, the choice (1.10) for our conformal factor explains our choice of time orientation on the interval $(0,1]$ and shows that future timelike infinity is located at $t=0$.

For use below, we introduce the background Minkowski metric

$$
\eta^{i j}=-\frac{\Lambda}{3} \delta_{0}^{i} \delta_{0}^{j}+\delta_{I}^{i} \delta_{J}^{j} \delta^{I J}
$$

the densitized three-metric

$$
\mathfrak{g}^{I J}=\operatorname{det}\left(\check{g}_{K L}\right)^{\frac{1}{3}} g^{I J}
$$

where

$$
\left(\check{g}_{I J}\right)=\left(g^{I J}\right)^{-1},
$$

and the variable

$$
\mathfrak{q}=g^{00}-\eta^{00}-\frac{\Lambda}{9} \ln \left(\operatorname{det}\left(g^{P Q}\right)\right) .
$$

Remark 1.2. While our choices for the coordinates and conformal factor are ultimately justified by the fact that they work, motivation for these choices can be seen by observing that

$$
\left(g^{i j}, \Phi\right)=\left(\eta^{i j},-\ln (t)\right)
$$

is an exact solution to the vacuum conformal Einstein equations, i.e. (1.7) with $\rho=0$, that is a member of the FLRW family of solutions and locally conformal to de Sitter space.

With our notation and conventions fixed, we are now able to state our future non-linear stability result in the following theorem, which is the main result of this article. The proof is given in Section 3 ,

Theorem 1.3. Suppose $\Lambda>0, \epsilon>0,0<K \leq 1 / 3, k \in \mathbb{Z}_{\geq 3}, g_{0}^{i j} \in H^{k+1}\left(\mathbb{T}^{3}\right), g_{1}^{i j}, \rho_{0}, v_{I}^{0} \in H^{k}\left(\mathbb{T}^{3}\right)$, $\rho_{0}(x)>0$ for all $x \in \mathbb{T}^{3}$, and that the quadruple

$$
\left.\left(g^{i j}, \partial_{t} g^{i j}, \rho, v_{I}\right)\right|_{t=1}=\left(g_{0}^{i j}, g_{1}^{i j}, \rho_{0}, v_{I}^{0}\right)
$$

satisfies the constraint equations

$$
\left.\left(G^{i 0}-T^{i 0}\right)\right|_{t=1}=0, \quad \text { and }\left.\quad Z^{k}\right|_{t=1}=0 .
$$

Then there exists a $\delta>0$, such that if

$$
\left\|g_{0}^{i j}-\eta^{i j}\right\|_{H^{k+1}}+\left\|g_{1}^{i j}\right\|_{H^{k}}+\left\|\rho_{0}\right\|_{H^{k}}+\left\|v_{I}\right\|_{H^{k}}<\delta,
$$

then there exists a classical solution $g^{i j} \in C^{2}\left((0,1] \times \mathbb{T}^{3}\right), \rho, v_{I} \in C^{1}\left((0,1] \times \mathbb{T}^{3}\right)$ to the conformal EinsteinEuler equations given by (1.7) and (1.8) that satisfies the initial conditions (1.14), the gauge condition $Z^{k}=0$ in $(0,1] \times \mathbb{T}^{3}$, the regularity conditions

$$
g^{i j} \in C^{0}\left((0,1], H^{k+1}\left(\mathbb{T}^{3}\right)\right) \cap C^{0}\left([0,1], H^{k}\left(\mathbb{T}^{3}\right)\right) \cap C^{1}\left((0,1], H^{k}\left(\mathbb{T}^{3}\right)\right) \cap C^{1}\left([0,1], H^{k-1}\left(\mathbb{T}^{3}\right)\right)
$$

and

$$
\rho, v_{I} \in C^{0}\left((0,1], H^{k}\left(\mathbb{T}^{3}\right)\right) \cap C^{0}\left([0,1], H^{k-1}\left(\mathbb{T}^{3}\right)\right)
$$

and the bounds

$$
\frac{\Lambda}{6} \leq-g^{00}(t, x) \leq \frac{2 \Lambda}{3}, \quad \frac{3}{2 \Lambda} \leq-g_{00}(t, x) \leq \frac{6}{\Lambda}, \quad \frac{1}{2} \delta^{I J} \leq g^{I J}(t, x) \leq \frac{3}{2} \delta^{I J},
$$

and

$$
\sqrt{\frac{3}{2 \Lambda}} \leq v_{0}(t, x) \leq \sqrt{\frac{6}{\Lambda}}
$$

for all $(t, x) \in(0,1] \times \mathbb{T}^{3}$. 
Moreover, there exist $\sigma, \gamma^{j} \in H^{k-1}\left(\mathbb{T}^{3}\right)$ with $\sigma(x)>0$ for all $x \in \mathbb{T}^{3}$, such that the solution satisfies

$$
\begin{aligned}
\left\|g^{0 j}(t)-\left(\eta^{0 j}+t \gamma^{j}\right)\right\|_{H^{k-1}} & \lesssim t^{2-\epsilon}, \\
\left\|\partial_{t} g^{0 j}(t)-2 t^{-1}\left(g^{0 j}(t)-\eta^{0 j}\right)+\gamma^{j}\right\|_{H^{k-1}} & \lesssim t, \\
\left\|\partial_{t} g^{0 j}(t)-t^{-1}\left(g^{0 j}(t)-\eta^{0 j}\right)\right\|_{H^{k-1}}+\left\|\partial_{I} g^{0 j}(t)\right\|_{H^{k-1}} & \lesssim t^{1-\epsilon}, \\
\|\mathfrak{q}(t)-\mathfrak{q}(0)\|_{H^{k}}+\left\|\partial_{t} \mathfrak{q}(t)\right\|_{H^{k-1}} & \lesssim t, \\
\left\|\mathfrak{g}^{I J}(t)-\mathfrak{g}^{I J}(0)\right\|_{H^{k}}+\left\|\partial_{t} \mathfrak{g}^{I J}(t)\right\|_{H^{k-1}} & \lesssim t, \\
\left\|t^{-3(1+K)} \rho(t)-\sigma\right\|_{H^{k-1}} & \lesssim t+t^{\frac{2(1-3 K)}{(1+\epsilon)}},
\end{aligned}
$$

and

$$
\left\|v_{I}(t)-v_{I}(0)\right\|_{H^{k-1}} \lesssim t^{\frac{1-3 K}{(1+\epsilon)}}
$$

for all $t \in[0,1]$, where $v_{I}(0)=0$ if $K \neq 1 / 3$.

We conclude with a few remarks:

(i) For any specified $\delta>0$, an open set of initial data satisfying the constraint equations (1.15) and the condition (1.16) can be constructed using a variation of the method employed in [9, §3].

(ii) The FLRW family of solutions correspond to the solutions obtained from initial data (1.14) of the form

$$
\left(g_{0}^{i j}, g_{1}^{i j}, \rho_{0}, v_{I}^{0}\right)=\left(-a_{0} \delta_{0}^{i} \delta_{0}^{j}+b_{0} \delta_{I}^{i} \delta_{J}^{j} \delta^{I J},-a_{1} \delta_{0}^{i} \delta_{0}^{j}+b_{1} \delta_{I}^{i} \delta_{J}^{j} \delta^{I J}, c, 0\right)
$$

where the constants $a_{0}, b_{0}, c \in \mathbb{R}_{>0}$ and $a_{1}, b_{1} \in \mathbb{R}$ are chosen so that the constraint equations (1.15) are satisfied.

(iii) More detailed behaviour of the solution near $t=0$ can be obtained by using the estimates (1.17)(1.23) from Theorem 1.3 together with another application of Theorem B.1 from Appendix B

(iv) As discussed in Section 1.3 of [12, it should be possible using Ringström's patching argument from [11. to generalize the stability result contained in Theorem 1.3 to other spatial topologies.

\section{The Reduced Conformal Einstein-Euler equations}

As discussed briefly in the introduction, our proof of Theorem 1.3 relies on expressing the conformal Einstein-Euler equations, given by (1.7) and (1.8), as a symmetric hyperbolic system of the type analyzed in Appendix B. We achieve this through the use of a wave gauge defined by the vanishing of the vector field (1.9) in conjunction with a suitable transformation of the field variables. We present the details of this procedure in Sections 2.1 and 2.2

2.1. The reduced conformal Einstein equations. Following H. Friedrich's 4 generalization of the gauge reduction method of Y.Choquet-Bruhat [2, Ch. VI, §8] that, crucially, allows for gauge source functions, we replace the Einstein equations (1.7) by the gauge reduced version:

$$
\begin{aligned}
-2 R_{i j}+2 \nabla_{(i} Z_{j)}+ & A_{i j}{ }^{k} Z_{k}=-4 \nabla_{i} \nabla_{j} \Phi+4 \nabla_{i} \Phi \nabla_{j} \Phi \\
& -2\left[\square \Phi+2|\nabla \Phi|^{2}+\left(\frac{1-K}{2} \rho+\Lambda\right) e^{2 \Phi}\right] g_{i j}-2(1+K) e^{2 \Phi} \rho v_{i} v_{j}
\end{aligned}
$$

where

$$
A^{i j}{ }_{k}=-X^{(i} \delta_{k}^{j)}+Y^{(i} \delta_{k}^{j)}
$$

We will refer to these equations as the reduced conformal Einstein equations.

Since the gauge condition $Z^{k}=0$ propagates, we can, with out loss of generality, assume that it holds, or equivalently that

$$
X^{k}=-Y^{k}
$$

which, in turn, implies that

$$
\nabla_{i} \nabla^{i} t=Y^{0}=\frac{2}{t}\left(g^{00}+\frac{\Lambda}{3}\right) .
$$

A straightforward calculation using (1.10), (1.11), (2.1) and the identity

$$
\nabla^{(i} \chi^{j)}=-\frac{1}{2} \partial_{t} g^{i j} \quad\left(\chi^{j}=\delta_{0}^{j}\right)
$$


then shows that reduced Einstein equations are equivalent to

$$
\begin{aligned}
\left.-2 R^{i j}+2 \nabla^{(i} X^{j}\right)-X^{i} X^{j}=\frac{2 \Lambda}{3 t} \partial_{t} g^{i j}-\frac{4 \Lambda}{3 t^{2}} & \left(g^{00}+\frac{\Lambda}{3}\right) \delta_{0}^{i} \delta_{0}^{j}-\frac{4 \Lambda}{3 t^{2}} g^{0 K} \delta_{0}^{(i} \delta_{K}^{j)} \\
& -\frac{2}{t^{2}} g^{i j}\left(g^{00}+\frac{\Lambda}{3}\right)-(1-K) \frac{\rho}{t^{2}} g^{i j}-2(1+K) \frac{\rho}{t^{2}} v^{i} v^{j}
\end{aligned}
$$

which we note can be expressed as

$$
\begin{aligned}
-g^{k l} \partial_{k} \partial_{l} g^{i j}-Q^{i j}(g, \partial g)=\frac{2 \Lambda}{3 t} \partial_{t} g^{i j}-\frac{4 \Lambda}{3 t^{2}} & \left(g^{00}+\frac{\Lambda}{3}\right) \delta_{0}^{i} \delta_{0}^{j}-\frac{4 \Lambda}{3 t^{2}} g^{0 K} \delta_{0}^{(i} \delta_{K}^{j)} \\
& -\frac{2}{t^{2}} g^{i j}\left(g^{00}+\frac{\Lambda}{3}\right)-(1-K) \frac{\rho}{t^{2}} g^{i j}-2(1+K) \frac{\rho}{t^{2}} v^{i} v^{j}
\end{aligned}
$$

where the $Q^{i j}$ are quadratic in $\partial g=\left(\partial_{k} g^{i j}\right)$ and analytic in $g=\left(g^{i j}\right)$ on the region $\operatorname{defined}$ by $\operatorname{det}\left(g^{i j}\right)<0$.

The first step in transforming (2.3) into the desired form involves parameterizing the three-metric $g^{I J}$ using densitized spatial metric (1.12) and the determinant variable (1.13). Using the identities

$$
-g^{k l} \partial_{k} \partial_{l}\left(\mathfrak{g}^{I J}\right)=\operatorname{det}\left(\check{g}_{P Q}\right)^{\frac{1}{3}} \mathcal{L}_{L M}^{I J}\left(-g^{l k} \partial_{l} \partial_{k} g^{L M}\right)-g^{k l} \partial_{k}\left(\operatorname{det}\left(\check{g}_{P Q}\right)^{\frac{1}{3}} \mathcal{L}_{L M}^{I J}\right) \partial_{l} g^{L M}
$$

and

where

$$
\partial_{t} \mathfrak{g}^{I J}=\operatorname{det}\left(\check{g}_{P Q}\right)^{\frac{1}{3}} \mathcal{L}_{L M}^{I J} \partial_{t} g^{L M}
$$

we see from (2.3) that $\mathfrak{g}^{I J}$ and $\mathfrak{q}$ satisfy

$$
\mathcal{L}_{L M}^{I J}=\delta_{L}^{I} \delta_{M}^{J}-\frac{1}{3} \check{g}_{L M} g^{I J}
$$

$$
-g^{k l} \partial_{k} \partial_{l} \mathfrak{g}^{I J}-\tilde{Q}^{I J}(g, \partial g)=\frac{2 \Lambda}{3 t} \partial_{t} \mathfrak{g}^{I J}-2(1+K) \operatorname{det}\left(\check{g}_{P Q}\right)^{\frac{1}{3}} \mathcal{L}_{L M}^{I J} \frac{\rho}{t^{2}} v^{L} v^{M}
$$

and

$$
-g^{k l} \partial_{k} \partial_{l} \mathfrak{q}-\tilde{Q}(g, \partial g)=\frac{2 \Lambda}{3 t} \partial_{t} \mathfrak{q}-2\left(\frac{g^{00}+\frac{\Lambda}{3}}{t}\right)^{2}+(1-K)\left(\frac{\Lambda}{3}-g^{00}\right) \frac{\rho}{t^{2}}+2(1+K)\left(\frac{2 \Lambda}{9} \check{g}_{I J} v^{I} v^{J}-v^{0} v^{0}\right) \frac{\rho}{t^{2}},
$$

respectively, where $\tilde{Q}^{I J}$ and $\tilde{Q}$ are quadratic in $\partial g=\left(\partial_{k} g^{i j}\right)$ and analytic in $g=\left(g^{i j}\right)$ on the region defined by $\operatorname{det}\left(g^{I J}\right)>0$ and $g_{00}<0$.

We proceed by writing the wave equations (2.3) $(i=0),(2.4)$ and (2.5) in first order form using the following definitions:

$$
\begin{aligned}
\mathfrak{u}^{0 j} & =\frac{g^{0 j}-\eta^{0 j}}{2 t} \\
\mathfrak{u}_{0}^{0 j} & =\partial_{t} g^{0 j}-\frac{3\left(g^{0 j}-\eta^{0 j}\right)}{2 t}, \\
\mathfrak{u}_{I}^{0 j} & =\partial_{I} g^{0 j} \\
\mathfrak{u}^{I J} & =\mathfrak{g}^{I J}-\delta^{I J} \\
\mathfrak{u}_{k}^{I J} & =\partial_{k} \mathfrak{g}^{I J} \\
\mathfrak{u} & =\mathfrak{q},
\end{aligned}
$$

and

$$
\mathfrak{u}_{k}=\partial_{k} \mathfrak{q}
$$

In terms of theses first order variables, it is not difficult to verify that the wave equations (2.3) $(i=0)$, (2.4), and (2.5) are equivalent to the following systems of symmetric hyperbolic equations:

$$
\begin{aligned}
A^{k} \partial_{k}\left(\begin{array}{c}
\mathfrak{u}_{0}^{0 l} \\
\mathfrak{u}_{J}^{0 l} \\
\mathfrak{u}^{0 l}
\end{array}\right) & =\frac{1}{t} \mathcal{A} \mathbb{P}\left(\begin{array}{c}
\mathfrak{u}_{0}^{0 l} \\
\mathfrak{u}_{J}^{0 l} \\
\mathfrak{u}^{0 l}
\end{array}\right)+F, \\
A^{k} \partial_{k}\left(\begin{array}{c}
\mathfrak{u}_{0}^{L M} \\
\mathfrak{u}_{J}^{L M} \\
\mathfrak{u}^{L M}
\end{array}\right) & =-\frac{2 g^{00}}{t} \Pi\left(\begin{array}{c}
\mathfrak{u}_{0}^{L M} \\
\mathfrak{u}_{J}^{L M} \\
\mathfrak{u}^{L M}
\end{array}\right)+\mathfrak{F},
\end{aligned}
$$


and

$$
A^{k} \partial_{k}\left(\begin{array}{c}
\mathfrak{u}_{0} \\
\mathfrak{u}_{J} \\
\mathfrak{u}
\end{array}\right)=-\frac{2 g^{00}}{t} \Pi\left(\begin{array}{c}
\mathfrak{u}_{0} \\
\mathfrak{u}_{J} \\
\mathfrak{u}
\end{array}\right)+\mathfrak{f}
$$

where

$$
\begin{aligned}
A^{0} & =\left(\begin{array}{ccc}
-g^{00} & 0 & 0 \\
0 & g^{I J} & 0 \\
0 & 0 & -g^{00}
\end{array}\right) \\
A^{K} & =\left(\begin{array}{ccc}
-2 g^{0 K} & -g^{J K} & 0 \\
-g^{I K} & 0 & 0 \\
0 & 0 & 0
\end{array}\right) \\
\mathbb{P} & =\left(\begin{array}{ccc}
\frac{1}{2} & 0 & \frac{1}{2} \\
0 & \delta_{K}^{J} & 0 \\
\frac{1}{2} & 0 & \frac{1}{2}
\end{array}\right), \\
\mathcal{A} & =\left(\begin{array}{ccc}
-g^{00} & 0 & 0 \\
0 & \frac{3}{2} g^{I K} & 0 \\
0 & 0 & -g^{00}
\end{array}\right), \\
\Pi & =\left(\begin{array}{ccc}
1 & 0 & 0 \\
0 & 0 & 0 \\
0 & 0 & 0
\end{array}\right), \\
F & =\left(\begin{array}{cc}
Q^{0 l}+4 \mathfrak{u}^{00}\left(\mathfrak{u}_{0}^{0 l}+\mathfrak{u}^{0 l}\right)-8 \mathfrak{u}^{00} \mathfrak{u}^{0 l}+6 \mathfrak{u}^{0 I} \mathfrak{u}_{I}^{0 l}-(1-K) \frac{\rho}{t^{2}} g^{0 l}-2(1+K) \frac{\rho}{t^{2}} v^{0} v^{l} \\
0
\end{array}\right) \\
\mathfrak{F} & =\left(\begin{array}{cc}
\tilde{Q}^{L M}+4 \mathfrak{u}^{00} \mathfrak{u}_{0}^{L M}-2(1+K) \operatorname{det}\left(\check{g}_{P Q}\right)^{\frac{1}{3}} \mathcal{L}_{R S}^{L M} \frac{\rho}{t^{2}} v^{R} v^{S} \\
0 & -g^{00} \mathfrak{u}_{0}^{L M}
\end{array}\right)
\end{aligned}
$$

and

$$
\mathfrak{f}=\left(\begin{array}{c}
\tilde{Q}+4 \mathfrak{u}^{00} \mathfrak{u}_{0}-8\left(\mathfrak{u}^{00}\right)^{2}+(1-K)\left(\frac{\Lambda}{3}-g^{00}\right) \frac{\rho}{t^{2}}+2(1+K)\left(\frac{2 \Lambda}{9} \check{g}_{R S} v^{R} v^{S}-v^{0} v^{0}\right) \frac{\rho}{t^{2}} \\
0 \\
-g^{00} \mathfrak{u}_{0}^{L M}
\end{array}\right)
$$

It is important to note that the term $\frac{\rho}{t^{2}}$ that appears in (2.21)-(2.23) is not a singular term. This is because we can bound the density variable $\zeta$ defined by (2.25) in the following section. Expressing $\frac{\rho}{t^{2}}$ in terms of $\zeta$, it is clear that this term is regular.

Another important point is that the first order variables (2.6)-(2.12) are completely equivalent for $t>0$ to the metric $g^{i j}$ and its first derivatives $\partial_{k} g^{i j}$. Consequently, we lose nothing by parameterizing the gravitational field in this fashion, and gain by having transformed the reduced conformal equations into a symmetric hyperbolic system of the form 1$]$ considered in Appendix B.

2.2. The conformal Euler equations. For the conformal Euler equations (1.8), we use the formulation from [10, §2.2]; see, in particular, equation (2.55) of that article. In this formulation, given the conformal factor (1.10) and the linear equation of state (1.4), the conformal Euler equations (1.8) are from the computations presented in [10, §2.2] readily seen to be given by

$$
B^{k} \partial_{k}\left(\begin{array}{c}
\zeta \\
v_{J}
\end{array}\right)=\frac{1}{t} \mathcal{B} \pi\left(\begin{array}{c}
\zeta \\
v_{J}
\end{array}\right)-H
$$

\footnotetext{
${ }^{1}$ To be precise, we still need to make the trivial coordinate transformation $t \mapsto-t$ to obtain the form considered in Appendix B
} 
where

$$
\begin{aligned}
\rho & =t^{3(1+K)} \rho_{c} e^{(1+K) \zeta}, \quad \rho_{c} \in \mathbb{R}_{>0}, \\
v_{0} & =v_{0}\left(g^{k l}, v_{L}\right):=\frac{-g^{0 I} v_{I}-\sqrt{\left(g^{0 I} v_{I}\right)^{2}-g^{00}\left(g^{I J} v_{I} v_{J}+1\right)}}{g^{00}}, \\
v^{i} & =v^{i}\left(g^{k l}, v_{L}\right):=g^{i J} v_{J}+g^{i 0} v_{0}\left(g^{k l}, v_{L}\right), \\
V^{I J} & :=\frac{\partial v^{I}}{\partial v_{J}}=g^{I J}-g^{I 0} \frac{v^{J}}{v^{0}}, \\
\mathcal{B} & =\frac{-1}{v^{0}}\left(\begin{array}{cc}
1 & 0 \\
0 & \frac{1-3 K}{v_{0}} V^{J I}
\end{array}\right), \\
\pi & =\left(\begin{array}{cc}
0 & 0 \\
0 & \delta_{I}^{J}
\end{array}\right), \\
L_{I}^{k} & =\delta_{J}^{k}-\frac{v_{J}}{v_{0}} \delta_{0}^{k}, \\
M_{I J} & =g_{I J}-\frac{v_{I}}{v_{0}} g_{0 J}-\frac{v_{J}}{v_{0}} g_{I 0}+\frac{g_{00}}{\left(v_{0}\right)^{2}} v_{I} v_{J}, \\
B^{0} & =\left(\begin{array}{cc}
K & \frac{K}{v^{0}} L_{M}^{0} V^{M J} \\
\frac{K}{v^{0}} V^{L I} L_{L}^{0} & V^{L I} M_{L M} V^{M J}
\end{array}\right), \\
B^{K} & =\frac{1}{v^{0}}\left(\begin{array}{cc}
K v^{K} & K L_{M}^{K} V^{M J} \\
K V^{L I} L_{L}^{K} & V^{L I} M_{L M} V^{M J} v^{K}
\end{array}\right),
\end{aligned}
$$

and

$$
H=-\frac{1}{v^{0}}\left(\begin{array}{c}
-K L_{L}^{k} \Gamma_{k l}^{L} v^{l}-K L_{L}^{k} \frac{\partial v^{L}}{\partial g^{l m}} \partial_{k} g^{l m} \\
-v^{L I} M_{L M} v^{k} \Gamma_{k l}^{M} v^{l}-V^{L I} M_{L M} v^{k} \frac{\partial v^{L}}{\partial g^{l m}} \partial_{k} g^{l m}
\end{array}\right) .
$$

\section{Proof of Theorem 1.3}

We begin by fixing $\Lambda>0, k \in \mathbb{Z}_{\geq 3}, K \in(0,1 / 3], \epsilon>0$ and $R>0$. Assuming $g_{00}<0, g^{00}<0$ and $\operatorname{det}\left(g^{I J}\right)>0$, we then observe that the relations

$$
\mathbb{P} A^{0} \mathbb{P} \leq \mathbb{P} \mathcal{A} \mathbb{P} \quad \text { and } \quad \Pi A^{0} \Pi=\frac{1}{2}\left(-2 g^{00} \Pi\right)
$$

follow directly from (2.16), (2.18) and (2.20).

Next, setting

$$
\begin{gathered}
\mathbf{A}^{0}=\left(\begin{array}{ccc}
A^{0} & 0 & 0 \\
0 & A^{0} & 0 \\
0 & 0 & A^{0}
\end{array}\right), \\
\mathcal{A}=\left(\begin{array}{ccc}
\mathcal{A} & 0 & 0 \\
0 & -2 g^{00} \mathbb{I} & 0 \\
0 & 0 & -2 g^{00} \mathbb{I}
\end{array}\right), \\
\mathbb{P}=\left(\begin{array}{ccc}
\mathbb{P} & 0 & 0 \\
0 & \Pi & 0 \\
0 & 0 & \Pi
\end{array}\right),
\end{gathered}
$$

and

$$
\mathfrak{u}=\left(\mathfrak{u}^{0 l}, \mathfrak{u}_{J}^{0 l}, \mathfrak{u}^{0 l}, \mathfrak{u}_{0}^{L M}, \mathfrak{u}_{J}^{L M}, \mathfrak{u}^{L M}, \mathfrak{u}_{0}, \mathfrak{u}_{J}, \mathfrak{u}\right)^{\operatorname{tr}},
$$

a straightforward calculation shows that

$$
\left[\mathbf{A}^{0}, \mathbb{P}\right]=[\mathcal{A}, \mathbb{P}]=0
$$

while we see from (2.16) that

$$
\mathbf{A}^{0}=\mathbf{A}^{0}\left(t, t \mathfrak{u}, \underline{P}^{\perp} \mathfrak{u}\right)
$$

Using (3.2) and (3.3), we compute

$$
D_{\mathfrak{u}} \mathbf{A}^{0} \cdot\left(\mathbf{A}^{0}\right)^{-1} \mathcal{A} \mathbb{P} \mathfrak{u}=t\left[D_{2} \mathbf{A}^{0}\left(t, t \mathfrak{u}, \underline{\mathbb{P}}^{\perp} \mathfrak{u}\right) \cdot\left(\mathbf{A}^{0}\right)^{-1} \mathcal{A} \underline{\mathbb{P}} \mathfrak{u}\right] \quad\left(\underline{\mathbb{P}}^{\perp}:=\mathbb{I}-\underline{\mathbb{P}}\right) .
$$

Next, it is clear from (2.29) and (2.30) that

$$
[\mathcal{B}, \pi]=0,
$$


and that

$$
\pi \mathcal{B}=0, \quad \text { if } K=1 / 3 .
$$

Setting

$$
\xi=\left(\mathfrak{u}, \zeta, v_{J}\right),
$$

and evaluating $B^{0}$ and $\mathcal{B}$ at $\xi=0$, we find that

$$
\left.B^{0}\right|_{\xi=0}=\left(\begin{array}{cc}
K & 0 \\
0 & \delta_{I J}
\end{array}\right) \quad \text { and }\left.\quad \mathcal{B}\right|_{\xi=0}=\left(\begin{array}{cc}
\sqrt{\frac{3}{\Lambda}} & 0 \\
0 & 1-3 K
\end{array}\right) .
$$

From these expressions, it is clear that

$$
\left.\pi B^{0}\right|_{\xi=0} \pi=\left.\frac{1}{1-3 K} \pi \mathcal{B}\right|_{\xi=0}=\left(\delta^{I J}\right), \quad \text { if } K \in(0,1 / 3) .
$$

We also observe from (2.33) that

$$
\pi^{\perp} B^{0}(t, \xi) \pi^{\perp}=K \quad\left(\pi^{\perp}:=\mathbb{I}-\pi\right),
$$

which, in turn, implies after differentiating that

$$
\pi^{\perp}\left[D_{\xi} B^{0}(t, \xi) \cdot \delta \xi\right] \pi^{\perp}=0
$$

for all $(t, \xi)$ in the domain of definition of $B^{0}$ and all variations $\delta \xi$.

From the evolution equations (2.13), (2.14), (2.15), and (2.24), the relations (3.1), (3.2), (3.4), (3.5), (3.6), (3.7), and (3.8), and Remark B.2, it is clear after performing the trivial time-coordinate transformation $t \mapsto-t$ that $\xi$ satisfies a symmetric hyperbolic system of the type to which we can apply Theorem B.1 from Appendix B. Doing so guarantees the existence of a $\delta>0$ such that if

$$
\left.\left(g^{i j}, \partial_{t} g^{i j}, \zeta, v_{J}\right)\right|_{t=1}=\left.\left(g_{0}^{i j}, \partial_{t} g_{0}^{i j}, \zeta_{0}, v_{J}^{0}\right)\right|_{t=1} \in H^{k+1}\left(\mathbb{T}^{3}\right) \times H^{k}\left(\mathbb{T}^{3}\right) \times H^{k}\left(\mathbb{T}^{3}\right) \times H^{k}\left(\mathbb{T}^{3}\right)
$$

and

$$
\rho_{c} \in \mathbb{R}_{>0}
$$

is chosen so that $\rho_{c}$ and the corresponding initial data $\left.\xi\right|_{t=1}=\xi_{0}$, which can be computed via the formulas (1.12), (1.13), (2.6) -(2.12) and (2.25), are bounded by

$$
\left\|\xi_{0}\right\|_{H^{k}}+\rho_{c} \leq \delta
$$

then there exists a map

$$
\left.\xi \in C^{1}\left((0,1] \times \mathbb{T}^{3}\right]\right) \cap C^{0}\left((0,1], H^{k}\left(\mathbb{T}^{3}\right)\right) \cap C^{0}\left([0,1], H^{k-1}\left(\mathbb{T}^{3}\right)\right)
$$

such that

$$
\|\xi\|_{L^{\infty}\left([0,1], H^{k}\right)} \leq R
$$

$\xi$ is the unique classical solution to (2.13), (2.14), (2.15) and (2.24) on $(0,1] \times \mathbb{T}^{3}$ that agrees with the initial data at $t=1$, i.e. $\left.\xi\right|_{t=1}=\xi_{0}$, the metric $g^{i j}$ and conformal four-velocity component $v_{0}$, as determined by the formulas (1.12), (1.13), (2.6), (2.9), (2.11) and (2.26), satisfy

$$
\frac{\Lambda}{6} \leq-g^{00}(t, x) \leq \frac{2 \Lambda}{3}, \quad \frac{3}{2 \Lambda} \leq-g_{00}(t, x) \leq \frac{6}{\Lambda}, \quad \frac{1}{2} \delta^{I J} \leq g^{I J}(t, x) \leq \frac{3}{2} \delta^{I J},
$$

and

$$
\sqrt{\frac{3}{2 \Lambda}} \leq v_{0}(t, x) \leq \sqrt{\frac{6}{\Lambda}}
$$

for all $(t, x) \in(0,1] \times \mathbb{T}^{3}$. Moreover, for given $\epsilon>0$, it follows, by choosing $R>0$ small enough, that the components of $\xi$ satisfy:

$$
\begin{aligned}
\left\|\mathfrak{u}_{0}^{0 j}(t)-\mathfrak{u}^{0 j}(t)+2 \mathfrak{u}^{0 j}(0)\right\|_{H^{k-1}} & \lesssim t, \\
\left\|\mathfrak{u}_{0}^{0 j}(t)+\mathfrak{u}^{0 j}(t)\right\|_{H^{k-1}}+\left\|\mathfrak{u}_{I}^{0 j}\right\|_{H^{k-1}} & \lesssim t^{1-\epsilon} \\
\left\|\mathfrak{u}^{I J}(t)-\mathfrak{u}^{I J}(0)\right\|_{H^{k-1}}+\left\|\mathfrak{u}_{K}^{I J}(t)-\mathfrak{u}_{K}^{I J}(0)\right\|_{H^{k-1}}+\left\|\mathfrak{u}_{0}^{I J}(t)\right\|_{H^{k-1}} & \lesssim t \\
\|\mathfrak{u}(t)-\mathfrak{u}(0)\|_{H^{k-1}}+\left\|\mathfrak{u}_{I}(t)-\mathfrak{u}_{I}(0)\right\|_{H^{k-1}}+\left\|\partial_{t} \mathfrak{u}(t)\right\|_{H^{k-1}} & \lesssim t \\
\|\zeta(t)-\zeta(0)\|_{H^{k-1}} & \lesssim t+t^{\frac{2(1-3 K)}{(1+\epsilon)}}
\end{aligned}
$$


and

$$
\left\|v_{I}(t)-v_{I}(0)\right\|_{H^{k-1}} \lesssim t^{\frac{1-3 K}{(1+\epsilon)}}
$$

for $0 \leq t \leq 1$, where

$$
v_{I}(0)=0 \quad \text { if } K \in(0,1 / 3) .
$$

Using (3.10)-(3.15), it follows directly from the formulas (1.12), (1.13), (2.6)-(2.12), and (2.25) that the metric and fluid density satisfy:

$$
\begin{aligned}
\left\|g^{0 j}(t)-\left(\eta^{0 j}+t \gamma^{j}\right)\right\|_{H^{k-1}} & \lesssim t^{2-\epsilon} \\
\left\|\partial_{t} g^{0 j}(t)-2 t^{-1}\left(g^{0 j}(t)-\eta^{0 j}\right)+\gamma^{j}\right\|_{H^{k-1}} & \lesssim t \\
\left\|\partial_{t} g^{0 j}(t)-t^{-1}\left(g^{0 j}(t)-\eta^{0 j}\right)\right\|_{H^{k-1}}+\left\|\partial_{I} g^{0 j}\right\|_{H^{k-1}} & \lesssim t^{1-\epsilon} \\
\|\mathfrak{q}(t)-\mathfrak{q}(0)\|_{H^{k}}+\left\|\partial_{t} \mathfrak{q}(t)\right\|_{H^{k-1}} & \lesssim t \\
\left\|\mathfrak{g}^{I J}(t)-\mathfrak{g}^{I J}(0)\right\|_{H^{k}}+\left\|\partial_{t} \mathfrak{g}^{I J}(t)\right\|_{H^{k-1}} & \lesssim t
\end{aligned}
$$

and

$$
\left\|t^{-3(1+K)} \rho(t)-\sigma\right\|_{H^{k-1}} \lesssim t+t^{\frac{2(1-3 K)}{(1+\epsilon)}},
$$

where $\sigma, \gamma^{j} \in H^{k-1}\left(\mathbb{T}^{3}\right)$, and $\sigma(x)>0$ for all $x \in \mathbb{T}^{3}$.

Since $\xi$ satisfies (2.13), (2.14),$(2.15)$ and (2.24) on $(0,1] \times \mathbb{T}^{3}$, it is clear from the results of Section 2.1 that the triple $\left(g^{i j}, \rho=t^{3(1+K)} \rho_{c} e^{(1+K) \zeta}, v_{I}\right)$ determined by $\xi$ solves the reduced conformal EinsteinEuler equations given by (1.8) and (2.2) on $(0,1] \times \mathbb{T}^{3}$. This fact together with the assumption that the initial data (3.9) satisfies the constraint equations (1.15) allows us to conclude via the Proposition on pg. 540 of [4] that the triple $\left(g^{i j}, \rho=t^{3(1+K)} \rho_{c} e^{(1+K) \zeta}, v_{I}\right)$ also satisfies the full conformal Einstein-Euler equations, given by (1.7) and (1.8), and the gauge condition $Z^{k}=0$ on $(0,1] \times \mathbb{T}^{3}$. This completes the proof of Theorem 1.3

Acknowledgements: This work was partially supported by the Australian Research Council grant FT1210045. I would like to thank C. Liu for useful discussions, and, in particular, for identifying an error in the statement and proof of a previous version of Theorem B.1. I also thank the referees for their comments and criticisms, which have served to improve the content and exposition of this article.

\section{Appendix A. Calculus inequalities}

In this appendix, we collect, for the convenience of the reader, a number of calculus inequalities that we employ throughout this article. The proof of the following inequalities are well known and may be found, for example, in the books [1], [3] and [14]. Before stating theses calculus inequalities, we first fix our notation and introduced a few definitions that will be needed in this appendix and the following one:

Coordinates, indexing and derivatives: Lower case Latin indices, e.g. $i, j, k$, will run from 0 to $n$, while upper case Latin indices, e.g. $I, J, K$, will run from 1 to $n$. We use $x=\left(x^{I}\right)$ to denote the standard periodic coordinates on the $n$-Torus $\mathbb{T}^{n}$ and $x^{0}=t$ to denote the time coordinate on intervals of the form $\left[T_{0}, T_{1}\right)$ where $T_{0}<T_{1} \leq 0$. Furthermore, we use lower case Greek letters to denote multi-indices, e.g. $\alpha=\left(\alpha_{1}, \alpha_{2}, \ldots, \alpha_{n}\right) \in \mathbb{Z}_{\geq 0}^{n}$, and employ the standard notation $D^{\alpha}=\partial_{1}^{\alpha_{1}} \partial_{2}^{\alpha_{2}} \cdots \partial_{n}^{\alpha_{n}}$ for spatial partial derivatives.

Inner-products and matrix inequalities: We employ the notation

$$
(\xi \mid \zeta)=\xi^{T} \zeta, \quad \xi, \zeta \in \mathbb{R}^{N}
$$

for the Euclidean inner-product on $\mathbb{R}^{N}$, and we denote the $L^{2}$ inner-product on $\mathbb{T}^{n}$ by

$$
\langle u \mid v\rangle=\int_{\mathbb{T}^{n}}(u(x) \mid v(x)) d^{n} x .
$$

For matrices $A, B \in \mathbb{M}_{N \times N}$, we define

$$
A \leq B \quad \Longleftrightarrow \quad(\xi \mid A \xi) \leq(\xi \mid B \xi), \quad \forall \xi \in \mathbb{R}^{N} .
$$

Constants and inequalities: We use that standard notation

$$
a \lesssim b
$$


for inequalities of the form

$$
a \leq C b
$$

in situations where the precise value or dependence on other quantities of the constant $C$ is not required. On the other hand, when the dependence of the constant on other inequalities needs to be specified, for example if the constant depends on the norm $\|u\|_{L^{\infty}}$, we use the notation

$$
C=C\left(\|u\|_{L^{\infty}}\right)
$$

Constants of this type will always be non-negative, non-decreasing, continuous functions of their arguments.

With the preliminaries out of the way, we now state the calculus inequalities:

Theorem A.1. [Hölder's inequality] If $0<p, q, r \leq \infty$ satisfy $1 / p+1 / q=1 / r$, then

$$
\|u v\|_{L^{r}} \leq\|u\|_{L^{p}}\|v\|_{L^{q}}
$$

for all $u \in L^{p}\left(\mathbb{T}^{n}\right)$ and $v \in L^{q}\left(\mathbb{T}^{n}\right)$.

Theorem A.2. [Sobolev's inequality] Suppose $1 \leq p<\infty$ and $s \in \mathbb{Z}_{>n / p}$. Then

$$
\|u\|_{L^{\infty}} \lesssim\|u\|_{W^{s, p}}
$$

for all $u \in W^{s, p}\left(\mathbb{T}^{n}\right)$.

Theorem A.3. [Product and commutator estimates]

(i) Suppose $1 \leq p_{1}, p_{2}, q_{1}, q_{2} \leq \infty, s=|\alpha| \in \mathbb{Z}_{\geq 1}$, and

$$
\frac{1}{p_{1}}+\frac{1}{p_{2}}=\frac{1}{q_{1}}+\frac{1}{q_{2}}=\frac{1}{r} .
$$

Then 2

$$
\left\|D^{\alpha}(u v)\right\|_{L^{r}} \lesssim\left\|D^{s} u\right\|_{L^{p_{1}}}\|v\|_{L^{q_{1}}}+\|u\|_{L^{p_{2}}}\left\|D^{s} v\right\|_{L^{q_{2}}}
$$

and

$$
\left\|D^{\alpha}(u v)-u D^{\alpha} v\right\|_{L^{r}} \lesssim\|D u\|_{L^{p_{1}}}\|v\|_{W^{s-1, q_{1}}}+\|D u\|_{W^{s-1, p_{2}}}\|v\|_{L^{q_{2}}}
$$

for all $u, v \in C^{\infty}\left(\mathbb{T}^{n}\right)$.

(ii) If $s_{1}, s_{2}, s_{3} \in \mathbb{Z}_{\geq 0}, s_{1}, s_{2} \geq s_{3}, 1 \leq p \leq \infty$, and $s_{1}+s_{2}-s_{3}>n / p$, then

$$
\|u v\|_{W^{s_{3}, p}} \lesssim\|u\|_{W^{s_{1}, p}}\|v\|_{W^{s_{2}, p}}
$$

for all $u \in W^{s_{1}, p}\left(\mathbb{T}^{n}\right)$ and $v \in W^{s_{2}, p}\left(\mathbb{T}^{n}\right)$.

Theorem A.4. [Moser's estimates] Suppose $s \in \mathbb{Z}_{\geq 1}, 1 \leq p \leq \infty,|\alpha| \leq s, f \in C^{s}(\mathbb{R}), f(0)=0$, and $V$ is open and bounded in $\mathbb{R}$. Then

$$
\left\|D^{\alpha} f(u)\right\|_{L^{p}} \leq C\left(\|f\|_{C^{s}(\bar{V})}\right)\left(1+\|u\|_{L^{\infty}}^{s-1}\right)\|u\|_{W^{s, p}}
$$

for all $u \in C^{0}\left(\mathbb{T}^{n}\right) \cap L^{\infty}\left(\mathbb{T}^{n}\right) \cap W^{s, p}\left(\mathbb{T}^{n}\right)$ with $u(\mathbf{x}) \in V$ for all $\mathbf{x} \in \mathbb{T}^{n}$.

Lemma A.5. [Ehrling's lemma] Suppose $1 \leq p<\infty, s_{0}, s, s_{1} \in \mathbb{Z}_{\geq 0}$, and $s_{0}<s<s_{1}$. Then for any $\epsilon>0$ there exists a constant $C=C(\epsilon)$ such that

$$
\|u\|_{W^{s, p}} \leq \epsilon\|u\|_{W^{s_{1}, p}}+C(\epsilon)\|u\|_{W^{s_{0}, p}}
$$

for all $u \in W^{s_{1}, p}\left(\mathbb{T}^{n}\right)$.

\footnotetext{
${ }^{2}$ Here, we are using the standard notation $\left\|D^{s} u\right\|_{L^{p}}^{p}=\sum_{|\alpha|=s}\left\|D^{\alpha} u\right\|_{L^{p}}^{p}$.
} 


\section{Appendix B. Symmetric hyperbolic Systems}

In this appendix, we analyze the initial value problem for symmetric hyperbolic systems of the form:

$$
\begin{aligned}
B^{i} \partial_{i} u & =\frac{1}{t} \mathcal{B P} u+H+G & & \text { in }\left[T_{0}, T_{1}\right) \times \mathbb{T}^{n}, \\
u & =u_{0} & & \text { in }\left\{T_{0}\right\} \times \mathbb{T}^{n},
\end{aligned}
$$

where

(i) $T_{0}<T_{1} \leq 0$,

(ii) $\mathbb{P}$ is a constant, symmetric projection operator, i.e. $\mathbb{P}^{2}=\mathbb{P}, \mathbb{P}^{T}=\mathbb{P}$ and $\partial_{i} \mathbb{P}=0$,

(iii) $v=v(t, x)$ is a $\mathbb{R}^{M}$-valued map, $u=u(t, x), G(t, x)$ and $H=H(t, x, v, u)$ are $\mathbb{R}^{N}$-valued maps, $H \in C^{0}\left(\left[T_{0}, 0\right], C^{\infty}\left(\mathbb{T}^{n} \times \mathbb{R}^{M} \times \mathbb{R}^{N}\right)\right)$ and satisfies $H(t, x, v, 0)=0$,

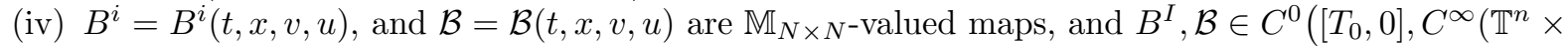
$\left.\left.\mathbb{R}^{M} \times \mathbb{R}^{N}\right)\right), B^{0} \in C^{1}\left(\left[T_{0}, 0\right], C^{\infty}\left(\mathbb{T}^{n} \times \mathbb{R}^{M} \times \mathbb{R}^{N}\right)\right)$ and they satisfy

$$
\left(B^{i}\right)^{T}=B^{i} \quad \text { and } \quad[\mathbb{P}, \mathcal{B}]=0,
$$

(v) there exists constants $\kappa, \gamma_{1}, \gamma_{2}>0$ such that

$$
\frac{1}{\gamma_{1}} \mathbb{I} \leq B^{0}(t, x, v, u) \leq \frac{1}{\kappa} \mathcal{B}(t, x, v, u) \leq \gamma_{2} \mathbb{I}
$$

(vi)

for all $(t, x, v, u) \in\left[T_{0}, 0\right] \times \mathbb{T}^{n} \times \mathbb{R}^{M} \times \mathbb{R}^{N}$,

$$
\mathbb{P}^{\perp} B^{0}\left(t, x, v, \mathbb{P}^{\perp} u\right) \mathbb{P}=\mathbb{P} B^{0}\left(t, x, v, \mathbb{P}^{\perp} u\right) \mathbb{P}^{\perp}=0
$$

for all $(t, x, v, u) \in\left[T_{0}, 0\right] \times \mathbb{T}^{n} \times \mathbb{R}^{M} \times \mathbb{R}^{N}$ where

$$
\mathbb{P}^{\perp}=\mathbb{I}-\mathbb{P}
$$

is the complementary projection operator 3

(vii) and there exists constants $\theta, \beta_{1}, \beta_{2}, \beta_{3}, \beta_{4} \geq 0$ and $\omega>0$ such that

$$
\begin{gathered}
\left|\mathbb{P}^{\perp}\left[D_{u} B^{0}(t, x, v, u) \cdot\left(B^{0}(t, x, v, u)\right)^{-1} \mathcal{B}(t, x, v, u) \mathbb{P} u\right] \mathbb{P}^{\perp}\right|_{\mathrm{op}} \leq|t| \theta+\frac{2 \beta_{1}}{\omega+\left|\mathbb{P}^{\perp} u\right|^{2}}|\mathbb{P} u|^{2}, \\
\left|\mathbb{P}^{\perp}\left[D_{u} B^{0}(t, x, v, u) \cdot\left(B^{0}(t, x, v, u)\right)^{-1} \mathcal{B}(t, x, v, u) \mathbb{P} u\right] \mathbb{P}\right|_{\mathrm{op}} \leq|t| \theta+\frac{2 \beta_{2}}{\sqrt{\omega+\left|\mathbb{P}^{\perp} u\right|^{2}}}|\mathbb{P} u|, \\
\left|\mathbb{P}\left[D_{u} B^{0}(t, x, v, u) \cdot\left(B^{0}(t, x, v, u)\right)^{-1} \mathcal{B}(t, x, v, u) \mathbb{P} u\right] \mathbb{P}^{\perp}\right|_{\mathrm{op}} \leq|t| \theta+\frac{2 \beta_{3}}{\sqrt{\omega+\left|\mathbb{P}^{\perp} u\right|^{2}}}|\mathbb{P} u|,
\end{gathered}
$$

and

$$
\left|\mathbb{P}\left[D_{u} B^{0}(t, x, v, u) \cdot\left(B^{0}(t, x, v, u)\right)^{-1} \mathcal{B}(t, x, v, u) \mathbb{P} u\right] \mathbb{P}\right|_{\text {op }} \leq|t| \theta+\beta_{4}
$$

for all $(t, x, v, u) \in\left[T_{0}, 0\right] \times \mathbb{T}^{n} \times \mathbb{R}^{M} \times \mathbb{R}^{N}$.

Before proceeding, we make some observations. First, we note that the bound (B.3) implies that $B^{0}$ and $\mathcal{B}$ are invertible and satisfy the matrix operator bounds

$$
\left|\left(B^{0}(t, x, v, u)\right)^{-1}\right|_{\mathrm{op}} \leq \gamma_{1} \quad \text { and } \quad\left|\mathcal{B}(t, x, v, u)^{-1}\right|_{\mathrm{op}} \leq \frac{\gamma_{1}}{\kappa}
$$

for all $(t, x, v, u) \in\left[T_{0}, 0\right] \times \mathbb{T}^{n} \times \mathbb{R}^{M} \times \mathbb{R}^{N}$. Second, a straightforward computation shows that the condition (B.4) is equivalent to

$$
\mathbb{P} B^{0}\left(t, x, v, \mathbb{P}^{\perp} u\right)^{-1} \mathbb{P}^{\perp}=\mathbb{P}^{\perp} B^{0}\left(t, x, v, \mathbb{P}^{\perp} u\right)^{-1} \mathbb{P}=0
$$

for all $(t, x, u) \in\left[T_{0}, 0\right] \times \mathbb{T}^{n} \times \mathbb{R}^{N}$. We also note that it follows immediately from the bounds (B.3) that there exists constants $\tilde{\kappa}, \tilde{\gamma}_{1}, \tilde{\gamma}_{2}>0$, where $\tilde{\gamma}_{1} \leq \gamma_{1}, \kappa \leq \tilde{\kappa}$ and $\tilde{\gamma}_{2} \leq \gamma_{2}$, such that

$$
\frac{1}{\tilde{\gamma}_{1}} \mathbb{P} \leq \mathbb{P} B^{0}(t, x, v, u) \mathbb{P} \leq \frac{1}{\tilde{\kappa}} \mathbb{P} \mathcal{B}(t, x, v, u) \mathbb{P} \leq \tilde{\gamma}_{2} \mathbb{P}
$$

for all $(t, x, v, u) \in\left[T_{0}, 0\right] \times \mathbb{T}^{n} \times \mathbb{R}^{M} \times \mathbb{R}^{N}$.

\footnotetext{
${ }^{3}$ In other words, $\mathbb{P}^{\perp} B^{0}(t, x, v, u) \mathbb{P}=\mathbb{P}^{\perp}\left[\tilde{B}^{0}(t, x, v, u) \cdot \mathbb{P} u\right] \mathbb{P}$ and $\mathbb{P} B^{0}(t, x, v, u) \mathbb{P}^{\perp}=\mathbb{P}\left[\hat{B}^{0}(t, x, v, u) \cdot \mathbb{P} u\right] \mathbb{P}$ for matrixvalued maps $\tilde{B}^{0}(t, x, v, u)$ and $\hat{B}^{0}(t, x, v, u)$ with the same regularity as $B^{0}(t, x, v, u)$.
} 
Theorem B.1. Suppose $k \in \mathbb{Z}_{>n / 2+1}, u_{0} \in H^{k}\left(\mathbb{T}^{n}\right), v, G \in C^{0}\left(\left[T_{0}, 0\right], H^{k}\left(\mathbb{T}^{n}\right)\right)$, assumptions (i)-(vii) are fulfilled, and the constants $\left\{\beta, \tilde{\kappa}, \tilde{\gamma}_{1}\right\}$ satisfy $0 \leq \beta \tilde{\gamma}_{1}<\tilde{\kappa}$ where $\beta=\sum_{i=1}^{4} \beta_{4}$. Then there exists a $T_{*} \in\left(T_{0}, 0\right)$, and a unique classical solution $u \in C^{1}\left(\left[T_{0}, T_{*}\right) \times \mathbb{T}^{n}\right)$ that satisfies $u \in C^{0}\left(\left[T_{0}, T_{*}\right), H^{k}\right) \cap$ $C^{1}\left(\left[T_{0}, T_{*}\right), H^{k-1}\right)$, the energy estimate

$$
\|u(t)\|_{H^{k}}^{2}+\|G\|_{L^{\infty}\left(\left[T_{0}, 0\right), H^{k}\right)}^{2}-\int_{T_{0}}^{t} \frac{1}{\tau}\|\mathbb{P} u(\tau)\|_{H^{k}}^{2} \leq C e^{C\left(t-T_{0}\right)}\left(\left\|u\left(T_{0}\right)\right\|_{H^{k}}^{2}+\|G\|_{L^{\infty}\left(\left[T_{0}, 0\right), H^{k}\right)}^{2}\right)
$$

for $T_{0} \leq t<T_{*}$ where

$$
C=C\left(\|u\|_{L^{\infty}\left(\left[T_{0}, T_{*}\right), H^{k}\right)},\|v\|_{L^{\infty}\left(\left[T_{0}, 0\right), H^{k}\right)},\|G\|_{L^{\infty}\left(\left[T_{0}, 0\right), H^{k}\right)}^{2},\left\|\partial_{t} v\right\|_{L^{\infty}\left(\left[T_{0}, 0\right), H^{k-1}\right)}, \theta, \gamma_{1}, \gamma_{2}, \tilde{\kappa}, \beta, \omega\right),
$$

and can be uniquely continued to a larger time interval $\left[T_{0}, T^{*}\right)$ for some $T^{*} \in\left(T_{*}, 0\right)$ provided that $\|u\|_{L^{\infty}\left(\left[T_{0}, T_{*}\right), W^{1, \infty}\right)}<\infty$.

Moreover, for any $R>0$, there exists a

$$
\delta=\delta\left(R,\|v\|_{L^{\infty}\left(\left[T_{0}, 0\right), H^{k}\right)},\left\|\partial_{t} v\right\|_{L^{\infty}\left(\left[T_{0}, 0\right), H^{k-1}\right)}, \theta, \gamma_{1}, \gamma_{2}, \beta, \tilde{\kappa}, \omega\right)>0
$$

such that if $\left\|u_{0}\right\|_{H^{k}}+\|G\|_{L^{\infty}\left(\left[T_{0}, 0\right), H^{k}\right)} \leq \delta$, then the solution $u(t, x)$ exists on the time interval $\left[T_{0}, 0\right)$ and can be uniquely extended to $\left[T_{0}, 0\right]$ as an element of $C^{0}\left(\left[T_{0}, 0\right], H^{k-1}\right)$ satisfying

$$
\|u\|_{L^{\infty}\left(\left[T_{0}, 0\right], W^{1, \infty}\right)} \leq R
$$

and

$$
\begin{aligned}
&\|\mathbb{P} u(t)\|_{H^{k-1}} \lesssim\left\{\begin{array}{ll}
-t & \text { if } \tilde{\kappa}-\beta_{4} \tilde{\gamma}_{1}>1 \\
(-t)^{\tilde{\kappa}-\beta_{4}} \tilde{\gamma}_{1}-\sigma & \text { if } 0<\sigma<\tilde{\kappa}-\beta_{4} \tilde{\gamma}_{1} \leq 1
\end{array},\right. \\
&\left\|\mathbb{P}^{\perp} u(t)-\mathbb{P}^{\perp} u(0)\right\|_{H^{k-1}} \lesssim\left\{\begin{array}{ll}
-t & \text { if } \tilde{\kappa}-\beta_{4} \tilde{\gamma}_{1}>1 \text { or }\left[B^{0}, \mathbb{P}\right]=0 \\
-t+(-t)^{2\left(\tilde{\kappa}-\beta_{4} \tilde{\gamma}_{1}-\sigma\right)} & \text { if } 0<\sigma<\tilde{\kappa}-\beta_{4} \tilde{\gamma}_{1} \leq 1
\end{array},\right.
\end{aligned}
$$

for $T_{0} \leq t \leq 0$.

Proof. First, the existence, uniqueness and continuation statements follow from standard results; for example, see [14, Ch.16 §1]. Therefore given a solution $u=u(t, x)$ defined on the time interval $\left[T_{0}, T_{*}\right)$, $T_{*} \in\left(T_{0}, 1\right)$, to (B.1)-(B.2), we act on (B.1) on the left by $D^{\alpha} \mathcal{B}^{-1}$ to obtain

$$
B^{0} \partial_{t} D^{\alpha} u+B^{I} \partial_{I} D^{\alpha} u=\frac{1}{t} \mathcal{B} D^{\alpha} \mathbb{P} u-\mathcal{B}\left[D^{\alpha}, \mathcal{B}^{-1} B^{0}\right] \partial_{t} u-\mathcal{B}\left[D^{\alpha}, \mathcal{B}^{-1} B^{I}\right] \partial_{I} u+\mathcal{B} D^{\alpha}\left(\mathcal{B}^{-1}(H+G)\right) .
$$

Using (B.1), we can write this as

$$
\begin{aligned}
& B^{0} \partial_{t} D^{\alpha} u+B^{I} \partial_{I} D^{\alpha} u=\frac{1}{t}\left[\mathcal{B} D^{\alpha} \mathbb{P} u-\mathcal{B}\left[D^{\alpha}, \mathcal{B}^{-1} B^{0}\right]\left(B^{0}\right)^{-1} \mathcal{B P} u\right]+\mathcal{B}\left[D^{\alpha}, \mathcal{B}^{-1} B^{0}\right]\left(B^{0}\right)^{-1} B^{I} \partial_{I} u \\
&-\mathcal{B}\left[D^{\alpha}, \mathcal{B}^{-1} B^{0}\right]\left(B^{0}\right)^{-1}(H+G)-\mathcal{B}\left[D^{\alpha}, \mathcal{B}^{-1} B^{I}\right] \partial_{I} u+\mathcal{B} D^{\alpha}\left(\mathcal{B}^{-1}(H+G)\right) .
\end{aligned}
$$

As far as energy estimates are concerned, the only potential problematic term is the one with the coefficient $\frac{1}{t}$ that becomes singular in the limit $t \nearrow 0$. Since the rest of the terms can be estimated using well known techniques, again see [14, Ch.16 §1], we will only estimate this potentially singular term in any detail.

Setting $\alpha=0$ in (B.11), we obtain, in the usual fashion for symmetric hyperbolic systems, the energy estimate

$$
\frac{1}{2} \partial_{t}\left\langle u \mid B^{0} u\right\rangle=\frac{1}{t}\langle u \mid \mathcal{B P} u\rangle+\frac{1}{2}\left\langle u \mid \partial_{0} B u\right\rangle+\frac{1}{2}\langle u \mid \operatorname{div} B u\rangle+\langle u \mid H\rangle+\langle u \mid G\rangle
$$

where

$$
\operatorname{div} B=\partial_{I} B^{I}
$$

Defining the energy norm

$$
\|u\|_{s}^{2}=\sum_{|\alpha| \leq s}\left\langle D^{\alpha} u \mid B^{0} D^{\alpha} u\right\rangle
$$

we obtain from (B.12) and the bounds (B.3) and (B.10) the estimate

$$
\partial_{t}\|u\|_{0}^{2} \leq \frac{2 \tilde{\kappa}}{t}\|\mathbb{P} u\|_{0}^{2}+\left\langle u \mid \partial_{t} B^{0} u\right\rangle+\gamma_{1}\|\operatorname{div} B\|_{L^{\infty}}\|u\|_{0}^{2}+2 \sqrt{\gamma_{1}}\left(\|H\|_{L^{2}}+\|G\|_{L^{2}}\right)\|u\|_{0}, \quad T_{0} \leq t<T_{*} .
$$

Using (B.1), we can write

$$
\left\langle u \mid \partial_{t} B^{0} u\right\rangle=\left\langle u \mid \widetilde{\partial_{t} B^{0}} u\right\rangle+\frac{1}{t}\left\langle u \mid\left[D_{u} B^{0}(t, \cdot, v, u) \cdot\left(B^{0}(t, \cdot, v, u)\right)^{-1} \mathcal{B}(t, \cdot, v, u) \mathbb{P} u\right] u\right\rangle
$$


where

$$
\begin{aligned}
\widetilde{\partial_{t} B^{0}}=\left(\partial_{t} B^{0}\right)(t, x, v, u) & +D_{v} B^{0}(t, x, v, u) \cdot \partial_{t} v \\
& +D_{u} B^{0}(t, x, v, u) \cdot\left[\left(B^{0}(t, x, v, u)\right)^{-1}\left(-B^{I}(t, x, v, u) \partial_{I} u+H(t, x, v, u)+G(t, x)\right)\right] .
\end{aligned}
$$

Observing that

$$
\begin{aligned}
\left\langle u \mid\left[D_{u} B^{0} \cdot\left(B^{0}\right)^{-1} \mathcal{B P} u\right] u\right\rangle & =\left\langle\mathbb{P}^{\perp} u \mid \mathbb{P}^{\perp}\left[D_{u} B^{0} \cdot\left(B^{0}\right)^{-1} \mathcal{B} \mathbb{P} u\right] \mathbb{P}^{\perp} \mathbb{P}^{\perp} u\right\rangle+\left\langle\mathbb{P}^{\perp} u \mid \mathbb{P}^{\perp}\left[D_{u} B^{0} \cdot\left(B^{0}\right)^{-1} \mathcal{B P} u\right] \mathbb{P P} u\right\rangle \\
& +\left\langle\mathbb{P} u \mid \mathbb{P}\left[D_{u} B^{0} \cdot\left(B^{0}\right)^{-1} \mathcal{B} \mathbb{P} u\right] \mathbb{P}^{\perp} \mathbb{P}^{\perp} u\right\rangle+\left\langle\mathbb{P} u \mid \mathbb{P}\left[D_{u} B^{0} \cdot\left(B^{0}\right)^{-1} \mathcal{B} \mathbb{P} u\right] \mathbb{P P} u\right\rangle,
\end{aligned}
$$

we see from (B.5)-(B.8) that

$$
\left|\left\langle u \mid\left[D_{u} B^{0} \cdot\left(B^{0}\right)^{-1} \mathcal{B P} u\right] u\right\rangle\right| \leq 4|t| \theta\|u\|_{L^{2}}^{2}+\beta\|\mathbb{P} u\|_{L^{2}}^{2},
$$

where $\beta=\sum_{i=1}^{4} \beta_{i}$. From (B.13), (B.14) and ( $(\underline{B .15})$, we see with the help of (B.3) that

$$
\partial_{t}\|u\|_{0}^{2} \leq \frac{2\left(\tilde{\kappa}-\beta \tilde{\gamma}_{1}\right)}{t}\|\mathbb{P} u\|_{0}^{2}+\gamma_{1}\left(4 \theta+\left\|\widetilde{\partial_{t} B^{0}}\right\|_{L^{\infty}}+\|\operatorname{div} B\|_{L^{\infty}}\right)\|u\|_{0}^{2}+2 \sqrt{\gamma_{1}}\left(\|H\|_{L^{2}}+\|G\|_{L^{2}}\right)\|u\|_{0}
$$

for $T_{0} \leq t<T_{*}$.

Next, using $[\mathbb{P}, \mathcal{B}]=0$, we observe, for $|\alpha| \leq k$, that

$$
\begin{aligned}
& \left\langle D^{\alpha} u \mid \mathcal{B}\left[D^{\alpha}, \mathcal{B}^{-1} B^{0}\right]\left(B^{0}\right)^{-1} \mathcal{B} \mathbb{P} u\right\rangle=\left\langle D^{\alpha} \mathbb{P}^{\perp} u \mid \mathcal{B}\left[D^{\alpha}, \mathcal{B}^{-1} \mathbb{P}^{\perp} B^{0} \mathbb{P}\right]\left(B^{0}\right)^{-1} \mathcal{B P} u\right\rangle \\
& \quad+\left\langle D^{\alpha} \mathbb{P}^{\perp} u \mid \mathcal{B}\left[D^{\alpha}, \mathcal{B}^{-1} \mathbb{P}^{\perp} B^{0}\right] \mathbb{P}^{\perp}\left(B^{0}\right)^{-1} \mathbb{P} \mathcal{B} \mathbb{P} u\right\rangle+\left\langle D^{\alpha} \mathbb{P} u \mid \mathcal{B}\left[D^{\alpha}, \mathcal{B}^{-1} B^{0}\right]\left(B^{0}\right)^{-1} \mathcal{B} \mathbb{P} u\right\rangle
\end{aligned}
$$

Applying the Sobolev, commutator, product and Moser calculus inequalities, i.e. Theorems A.2 A.3 and A.4. to the above expression, we find with the help of (B.4) and (B.9) the estimate

$$
\left\langle D^{\alpha} u \mid \mathcal{B}\left[D^{\alpha}, \mathcal{B}^{-1} B^{0}\right]\left(B^{0}\right)^{-1} \mathcal{B P} u\right\rangle \leq C\left(K_{1}, K_{2}\right)\|\mathbb{P} u\|_{H^{k}}\|\mathbb{P} u\|_{H^{k-1}}
$$

where

$$
K_{1}=\|u\|_{L^{\infty}\left(\left[T_{0}, T_{*}\right), H^{k}\right)} \quad \text { and } \quad K_{2}=\|v\|_{L^{\infty}\left(\left[T_{0}, 0\right), H^{k}\right)} .
$$

Hence, for any $\epsilon>0$, we find that

$$
\left\langle D^{\alpha} u \mid \mathcal{B}\left[D^{\alpha}, \mathcal{B}^{-1} B^{0}\right]\left(B^{0}\right)^{-1} \mathcal{B P} u\right\rangle \leq C\left(K_{1}, K_{2}\right)\left(\epsilon\|\mathbb{P} u\|_{H^{k}}^{2}+c(\epsilon)\|\mathbb{P} u\|_{L^{2}}^{2}\right)
$$

by Ehrling's lemma, Lemma A.5 and Young's inequality in the form $a b \leq \frac{1}{2 r} a^{2}+\frac{r}{2} b^{2}$ for $a, b \geq 0$ and $r>0$. We also see from

$$
\begin{aligned}
& \left\langle D^{\alpha} u \mid\left[D_{u} B^{0} \cdot\left(B^{0}\right)^{-1} \mathcal{B P} u\right] D^{\alpha} u\right\rangle= \\
& \left\langle\mathbb{P}^{\perp} D^{\alpha} u \mid \mathbb{P}^{\perp}\left[D_{u} B^{0} \cdot\left(B^{0}\right)^{-1} \mathcal{B P} u\right] \mathbb{P}^{\perp} \mathbb{P}^{\perp} D^{\alpha} u\right\rangle+\left\langle\mathbb{P}^{\perp} D^{\alpha} u \mid \mathbb{P}^{\perp}\left[D_{u} B^{0} \cdot\left(B^{0}\right)^{-1} \mathcal{B P} u\right] \mathbb{P P} D^{\alpha} u\right\rangle \\
& \quad+\left\langle\mathbb{P} D^{\alpha} u \mid \mathbb{P}\left[D_{u} B^{0} \cdot\left(B^{0}\right)^{-1} \mathcal{B} \mathbb{P} u\right] \mathbb{P}^{\perp} \mathbb{P}^{\perp} D^{\alpha} u\right\rangle+\left\langle\mathbb{P} D^{\alpha} u \mid \mathbb{P}\left[D_{u} B^{0} \cdot\left(B^{0}\right)^{-1} \mathcal{B} \mathbb{P} u\right] \mathbb{P P} D^{\alpha} u\right\rangle,
\end{aligned}
$$

the bounds (B.5)-(B.8), and the calculus inequalities that

$$
\begin{aligned}
\mid\left\langle D^{\alpha} u\right|[ & \left.\left.D_{u} B^{0} \cdot\left(B^{0}\right)^{-1} \mathcal{B P} u\right] D^{\alpha} u\right\rangle|\leq 4| t \mid \theta\left\|D^{\alpha} u\right\|_{L^{2}}^{2} \\
& +C\left(K_{1}, \omega\right)\left(\beta_{1}\|\mathbb{P} u\|_{L^{2}}^{2}+\left(\beta_{2}+\beta_{3}\right)\|\mathbb{P} u\|_{L^{2}}\left\|\mathbb{P} D^{\alpha} u\right\|_{L^{2}}\right)+2 \beta_{4}\left\|\mathbb{P} D^{\alpha} u\right\|_{L^{2}}^{2} .
\end{aligned}
$$

Applying Young's inequality to the this expression, we see for any $\epsilon>0$ that $\left|\left\langle D^{\alpha} u \mid\left[D_{u} B^{0} \cdot\left(B^{0}\right)^{-1} \mathcal{B P} u\right] D^{\alpha} u\right\rangle\right| \leq 4|t| \theta\|u\|_{H^{k}}^{2}+C\left(K_{1}, \theta, \beta, \omega\right)\left(\epsilon\|\mathbb{P} u\|_{H^{k}}^{2}+c(\epsilon)\|\mathbb{P} u\|_{L^{2}}^{2}\right)+2 \beta\left\|\mathbb{P} D^{\alpha} u\right\|_{L^{2}}^{2}$.

Setting

$$
\nu=\|G\|_{L^{\infty}\left(\left[T_{0}, 0\right), H^{k}\right)}
$$

and applying the standard energy estimates, i.e. as in the derivation of (B.12), for symmetric hyperbolic systems to (B.11), we obtain, after summing over $|\alpha| \leq k$ and using (B.17) and (B.18) together with the calculus inequalities, the estimate 4

$$
\frac{1}{2} \partial_{t}\|u\|_{k}^{2} \leq \frac{\tilde{\kappa}-\beta \tilde{\gamma}}{t}\|\mathbb{P} u\|_{k}^{2}+C\left(K_{1}, K_{2}, K_{3}, \theta, \gamma_{1}, \beta, \omega, \nu\right)\left[-\frac{1}{t}\left(\epsilon\|\mathbb{P} u\|_{k}^{2}+c(\epsilon)\|\mathbb{P} u\|_{0}^{2}\right)+\|u\|_{k}^{2}+\nu\right]
$$

for $T_{0} \leq t<T_{*}$, where

$$
K_{3}=\left\|\partial_{t} v\right\|_{L^{\infty}\left(\left[T_{0}, 0\right), H^{k-1}\right)} .
$$

\footnotetext{
${ }^{4}$ Here, we are taking essentially the same approach to deriving energy estimates for symmetric hyperbolic systems as in 14. Ch.16 §1].
} 
Since $\tilde{\kappa}-\beta / \tilde{\gamma}_{1}>0$ by assumption, we see, after choosing $\epsilon$ small enough and adding a suitable multiple of (B.16) to (B.19), that

$$
\partial_{t}\left(\|u\|_{k}^{2}+c\|u\|_{0}^{2}+\nu^{2}-\int_{T_{0}}^{t} \frac{1}{\tau}\|\mathbb{P} u(\tau)\|_{k}^{2} d \tau\right) \leq C\left(K_{1}, K_{2}, K_{3}, \theta, \gamma_{1}, \beta, \omega, \nu\right)\left(\|u\|_{k}^{2}+\nu^{2}\right)
$$

where $c=c\left(K_{1}, K_{2}, K_{3}, \theta, \gamma_{1}, \beta, \tilde{\kappa}, \omega, \nu\right)$. Applying Gronwall's inequality yields

$$
\|u(t)\|_{H^{k}}^{2}+\nu^{2}-\int_{T_{0}}^{t} \frac{1}{\tau}\|\mathbb{P} u(\tau)\|_{H^{k}}^{2} \leq C e^{C\left(t-T_{0}\right)}\left(\left\|u\left(T_{0}\right)\right\|_{H^{k}}^{2}+\nu^{2}\right), \quad T_{0} \leq t<T_{*},
$$

for some constant $C\left(K_{1}, K_{2}, K_{3}, \theta, \gamma_{1}, \gamma_{2}, \beta, \tilde{\kappa}, \omega, \nu\right)$. From this, Sobolev's inequality and the continuation principle, it follows, for any fixed $R>0$, that there exists a

$$
\delta=\delta\left(R, K_{2}, K_{3}, \theta, \gamma_{1}, \gamma_{2}, \beta, \tilde{\kappa}, \omega\right)>0
$$

such that the solution $u=u(t, x)$ exists on the time interval $\left[T_{0}, 0\right)$ and satisfies

$$
\|u\|_{L^{\infty}\left(\left[T_{0}, 0\right), H^{k}\right)}+\nu+\left(-\int_{T_{0}}^{0} \frac{1}{\tau}\|\mathbb{P} u(\tau)\|_{H^{k}}^{2} d \tau\right)^{\frac{1}{2}} \leq R
$$

provided that $\left\|u\left(T_{0}\right)\right\|_{H^{k}}+\nu<\delta$.

To complete the proof, we assume that the condition $\left\|u\left(T_{0}\right)\right\|_{H^{k}}+\nu<\delta$ holds. Writing (B.1) as

$$
\partial_{t} u=\left(B^{0}\right)^{-1}\left(B^{I} \partial_{I} u+\frac{1}{t} \mathcal{B P} u+H+G\right)
$$

and multiplying on the left by $\mathbb{P}^{\perp}$, we obtain

$$
\partial_{t} \mathbb{P}^{\perp} u=\mathbb{P}^{\perp}\left(B^{0}\right)^{-1}\left(B^{I} \partial_{I} u+H+G\right)+\frac{1}{t} \mathbb{P}^{\perp}\left(B^{0}\right)^{-1} \mathbb{P} \mathcal{B} \mathbb{P} u
$$

Integrating this in time, we see after applying the $H^{k-1}$ norm that

$$
\begin{aligned}
& \left\|\mathbb{P}^{\perp} u\left(t_{2}\right)-\mathbb{P}^{\perp} u\left(t_{1}\right)\right\|_{H^{k-1}} \leq \int_{t_{1}}^{t_{2}}\left\|\mathbb{P}^{\perp}\left(B^{0}(\tau)\right)^{-1} B^{I}(\tau) \partial_{I} u(\tau)\right\|_{H^{k-1}} \\
& \quad+\left\|\mathbb{P}^{\perp}\left(B^{0}(\tau)\right)^{-1} H(\tau)\right\|_{H^{k-1}}+\left\|\mathbb{P}^{\perp}\left(B^{0}(\tau)\right)^{-1} G(\tau)\right\|_{H^{k-1}}-\frac{1}{\tau}\left\|\mathbb{P}^{\perp}\left(B^{0}(\tau)\right)^{-1} \mathbb{P} \mathcal{B} \mathbb{P} u(\tau)\right\|_{H^{k-1}} d \tau
\end{aligned}
$$

for any $t_{1}, t_{2}$ satisfying $T_{0} \leq t_{1}<t_{2}$. Using the calculus inequalities, i.e. product, Sobolev, Hölder, and Moser, in conjunction with (B.9) and the energy estimates (B.20), we obtain from the above inequality that

$\left\|\mathbb{P}^{\perp} u\left(t_{2}\right)-\mathbb{P}^{\perp} u\left(t_{1}\right)\right\|_{H^{k-1}} \leq C\left(R, K_{2}\right)\left(\left|t_{2}-t_{1}\right|-\int_{t_{t}}^{t_{2}} \frac{1}{\tau}\|\mathbb{P} u(\tau)\|_{H^{k-1}}^{2} d \tau\right) \leq C\left(R, K_{2}\right)\left(\left|t_{2}-t_{1}\right|+o\left(\left|t_{2}-t_{1}\right|\right)\right)$.

It follows directly from this inequality that $\lim _{t \nearrow_{0}} \mathbb{P}^{\perp} u(t)$ exists in $H^{k-1}\left(\mathbb{T}^{n}\right)$, and moreover, that

$$
\mathbb{P}^{\perp} u \in C^{0}\left(\left[T_{0}, 0\right], H^{k-1}\right) .
$$

Next, we apply the $H^{k-1}$ norm to (B.21) and then integrate in time. With the help of the calculus inequalities, (B.9), and (B.20), this yields the estimate

$$
\int_{T_{0}}^{t}\left\|\partial_{t} \mathbb{P}^{\perp} u(\tau)\right\|_{H^{k-1}} d \tau \leq C\left(R, K_{2}\right) \quad T_{0} \leq t<0 .
$$

Multiplying (B.1) on the left by $\mathbb{P}$ shows that $\mathbb{P} u$ satisfies

$$
\bar{B}^{i} \partial_{i} \mathbb{P} u=\frac{1}{t} \overline{\mathcal{B}} \mathbb{P} u+\bar{H}+\bar{G}
$$

where

$$
\bar{B}^{i}=\mathbb{P} B^{i} \mathbb{P}, \quad \overline{\mathcal{B}}=\mathbb{P} \mathcal{B P}, \quad \bar{G}=\mathbb{P} G
$$

and

$$
\bar{H}=\mathbb{P} H+\mathbb{P} B^{i} \mathbb{P}^{\perp} \partial_{i} \mathbb{P}^{\perp} u
$$


Applying the same arguments used to derive the energy estimate (B.13) to the equation (B.24) yields

$$
\frac{1}{2} \partial_{t}\|\mathbb{P} u\|_{0}^{2} \leq \frac{\tilde{\kappa}-\beta_{4} \tilde{\gamma}_{1}}{t}\|\mathbb{P} u\|_{0}^{2}+\frac{\tilde{\gamma}_{1}}{2}\left(\theta+\left\|\widetilde{\partial_{t} \bar{B}^{0}}\right\|_{L^{\infty}}+\|\operatorname{div} \bar{B}\|_{L^{\infty}}\right)\|\mathbb{P} u\|_{0}^{2}+\sqrt{\tilde{\gamma}_{1}}\left(\|\bar{H}\|_{L^{2}}+\|\bar{G}\|_{L^{2}}\right)\|\mathbb{P} u\|_{0}
$$

for $T_{0} \leq t<0$, where now the energy norm $\|\cdot\|$ is defined by

$$
\|u\|_{s}^{2}=\sum_{|\alpha| \leq s}\left\langle D^{\alpha} u \mid \bar{B}^{0} D^{\alpha} u\right\rangle,
$$

and

$$
\begin{aligned}
\widetilde{\partial_{t} \bar{B}^{0}}=\left(\partial_{t} \bar{B}^{0}\right)(t, x, v, u)+D_{v} \bar{B}^{0}(t, x, v, u) \cdot \partial_{t} v \\
\quad+D_{u} \bar{B}^{0}(t, x, v, u) \cdot\left[\left(B^{0}(t, x, v, u)\right)^{-1}\left(-B^{I}(t, x, v, u) \partial_{I} u+H(t, x, v, u)+G(t, x)\right)\right] .
\end{aligned}
$$

Using (B.4) and (B.20), we see that (B.25) implies that

$$
\partial_{t}\|\mathbb{P} u\|_{0} \leq \frac{\tilde{\kappa}-\beta_{4} \tilde{\gamma}_{1}}{t}\|\mathbb{P} u\|_{0}+C\left(R, K_{2}, K_{3}, \theta, \gamma_{1}, \nu\right)\left[\left(1+\left\|\partial_{t} \mathbb{P}^{\perp} u\right\|_{H^{k-1}}\right)\|\mathbb{P} u\|_{0}+\|u\|_{H^{1}}+\|\bar{G}\|_{L^{2}}\right]
$$

for $T_{0} \leq t<0$. It then follows from Gronwall's inequality 5 and the bounds (B.20) and (B.23) that

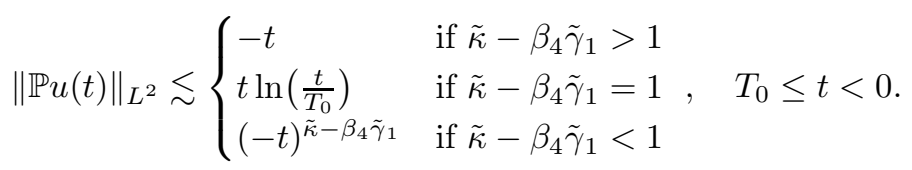

Proceeding as above, we can apply the same arguments used to derive the energy estimate (B.19) to (B.24) to obtain the estimate

$$
\frac{1}{2} \partial_{t}\|\mathbb{P} u\|_{k-1}^{2} \leq \frac{\tilde{\kappa}-\beta_{4} \tilde{\gamma}_{1}}{t}\|\mathbb{P} u\|_{k-1}^{2}+C\left(R, K_{2}, K_{3}, \theta, \gamma_{1}, \nu\right)\left[-\frac{1}{t}\left(\epsilon\|\mathbb{P} u\|_{k-1}^{2}+c(\epsilon)\|\mathbb{P} u\|_{0}^{2}\right)+\|\mathbb{P} u\|_{k-1}\right] .
$$

Choosing $\epsilon$ small enough, we see that the inequality

$$
\partial_{t}\|\mathbb{P} u\|_{k-1} \leq \frac{\tilde{\kappa}-\beta_{4} \tilde{\gamma}_{1}-\sigma}{t}\|\mathbb{P} u\|_{k-1}+C\left(R, K_{2}, K_{3}, \theta, \gamma_{1}, \gamma_{2}, \nu, \beta_{4}, \tilde{\kappa}\right)\left(\frac{1}{t}\|\mathbb{P} u\|_{L^{2}}+1\right)
$$

holds for any fixed $\sigma>0$. Choosing

$$
\sigma \in\left\{\begin{array}{ll}
\left(0, \tilde{\kappa}-\beta_{4} \tilde{\gamma}_{1}-1\right) & \text { if } \tilde{\kappa}-\beta_{4} \tilde{\gamma}_{1}>1 \\
\left(0, \tilde{\kappa}-\beta_{4} \tilde{\gamma}_{1}\right) & \text { if } \tilde{\kappa}-\beta_{4} \tilde{\gamma}_{1} \leq 1
\end{array},\right.
$$

it follows from (B.26) and an application of Gronwall's inequality to (B.27), that $\mathbb{P} u$ satisfies the decay estimate

$$
\|\mathbb{P} u(t)\|_{H^{k-1}} \lesssim\left\{\begin{array}{ll}
-t & \text { if } \tilde{\kappa}-\beta_{4} \tilde{\gamma}_{1}>1 \\
(-t)^{\tilde{\kappa}-\beta_{4} \tilde{\gamma}_{1}-\sigma} & \text { if } \tilde{\kappa}-\beta_{4} \tilde{\gamma}_{1} \leq 1
\end{array}, \quad T_{0} \leq t<0\right.
$$

Using the above estimate in conjunction with $(\overline{B .22})$, we see with the help of the calculus inequalities and the bound (B.20) that

$$
\left\|\mathbb{P}^{\perp} u\left(t_{2}\right)-\mathbb{P}^{\perp} u\left(t_{1}\right)\right\|_{H^{k-1}} \lesssim \begin{cases}\left|t_{2}-t_{1}\right| & \text { if } \tilde{\kappa}-\beta_{4} \tilde{\gamma}_{1}>1 \\ \left|t_{2}-t_{1}\right|+\left(-t_{1}\right)^{2\left(\tilde{\kappa}-\beta_{4} \tilde{\gamma}_{1}-\sigma\right)}-\left(-t_{2}\right)^{2\left(\tilde{\kappa}-\beta_{4} \tilde{\gamma}_{1}-\sigma\right)} & \text { if } \tilde{\kappa}-\beta_{4} \tilde{\gamma}_{1} \leq 1\end{cases}
$$

for any $t_{1}, t_{2}$ satisfying $T_{0} \leq t_{1}<t_{2}<0$. We also note in $(B .22)$ that if $\left[B^{0}, \mathbb{P}\right]=0$, then the term with the $\frac{1}{\tau}$ vanishes and we obtain the estimate

$$
\left\|\mathbb{P}^{\perp} u\left(t_{2}\right)-\mathbb{P}^{\perp} u\left(t_{1}\right)\right\|_{H^{k-1}} \leq C\left(R, K_{2}\right)\left|t_{2}-t_{1}\right|, \quad T_{0} \leq t_{1}<t_{2}<0 .
$$

\footnotetext{
${ }^{5}$ Here, we are using the following form of Gronwall's inequality: if $x(t)$ satisfies $x^{\prime}(t) \leq a(t) x(t)+h(t), t \geq T_{0}$, then $x(t) \leq x\left(T_{0}\right) e^{A(t)}+\int_{T_{0}}^{t} e^{A(t)-A(\tau)} h(\tau) d \tau$ where $A(t)=\int_{T_{0}}^{t} a(\tau) d \tau$. In particular, we observe from this that if $a(t)=\frac{\lambda}{t}+b(t), \lambda \in \mathbb{R}$, where $|b(t)| \leq r$ then

for $T_{0} \leq t<0$.

$$
x(t) \leq e^{r\left(t-T_{0}\right)} x\left(T_{0}\right)\left(\frac{t}{T_{0}}\right)^{\lambda}+e^{r\left(t-2 T_{0}\right)}(-t)^{\lambda} \int_{T_{0}}^{t} \frac{|h(\tau)|}{(-\tau)^{\lambda}} d \tau
$$
}


Sending $t_{2} \nearrow 0$ in $(\overline{\mathrm{B} .28})$ and $(\underline{\mathrm{B} .29})$, we see that $\mathbb{P}^{\perp} u$ satisfies

$$
\left\|\mathbb{P}^{\perp} u(t)-\mathbb{P}^{\perp} u(0)\right\|_{H^{k-1}} \lesssim \begin{cases}-t & \text { if } \tilde{\kappa}-\beta_{4} \gamma_{1}>1 \text { or }\left[B^{0}, \mathbb{P}\right]=0 \\ -t+(-t)^{2\left(\tilde{\kappa}-\beta_{4} \tilde{\gamma}_{1}-\sigma\right)} & \text { if } \tilde{\kappa}-\beta_{4} \tilde{\gamma}_{1} \leq 1\end{cases}
$$

for $T_{0} \leq t<0$.

\section{Remark B.2.}

(i) The bounds (B.3)-(B.8) and (B.10) do not need to hold for all $(v, u) \in \mathbb{R}^{M} \times \mathbb{R}^{N}$. The conclusions of Theorem B.1 remain valid provided that

(a) there exists constants $r, \mathcal{R}>0$ such that (B.3)-(B.8) and (B.10) hold for all $(t, x, v, u) \in$ $\left[T_{0}, 0\right] \times \mathbb{T}^{n} \times B_{r}\left(\mathbb{R}^{M}\right) \times B_{\mathcal{R}}\left(\mathbb{R}^{N}\right)$

(b) the map $v \in C^{0}\left(\left[T_{0}, 0\right], H^{k}\left(\mathbb{T}^{n}\right)\right)$ satisfies $\|v\|_{L^{\infty}\left(\left[T_{0}, 0\right] \times \mathbb{R}^{M}\right)}<r$,

(c) and the continuation principle is modified to having the solution $u(t, x)$ remain in a proper subset of $B_{\mathcal{R}}\left(\mathbb{R}^{N}\right)$ for $(t, x) \in\left[T_{0}, T_{*}\right) \times \mathbb{T}^{n}$ in addition to satisfying $\|u\|_{L^{\infty}\left(\left[T_{0}, T_{*}\right), W^{1, \infty}\right)}<\infty$,

(d) and the constant $R>0$ is chosen so that $R<\mathcal{R}$.

(ii) Given any two constants $\tilde{\beta}_{1}, \tilde{\beta}_{2}>0$, we observe that we can satisfy

$$
C_{1} \leq \frac{2 \beta_{1}}{\omega+\left|\mathbb{P}^{\perp} u\right|^{2}} \quad \text { and } \quad C_{2} \leq \frac{2 \beta_{2}}{\sqrt{\omega+\left|\mathbb{P}^{\perp} u\right|^{2}}}
$$

for all $u \in B_{\mathcal{R}}\left(\mathbb{R}^{N}\right)$ by setting

$$
\beta_{1}=\frac{\tilde{\beta}_{1}\left(\mathcal{R}+\mathcal{R}^{2}\right)}{2}, \quad \beta_{1}=\frac{\tilde{\beta}_{2} \sqrt{\left(\mathcal{R}+\mathcal{R}^{2}\right)}}{2} \quad \text { and } \quad \omega=\mathcal{R} .
$$

This shows that if the bounds

$$
\begin{gathered}
\left|\mathbb{P}^{\perp}\left[D_{u} B^{0}(t, x, v, u) \cdot\left(B^{0}(t, x, v, u)\right)^{-1} \mathcal{B}(t, x, v, u) \mathbb{P} u\right] \mathbb{P}^{\perp}\right|_{\mathrm{op}} \leq|t| \theta+\tilde{\beta}_{1}|\mathbb{P} u|^{2}, \\
\left|\mathbb{P}^{\perp}\left[D_{u} B^{0}(t, x, v, u) \cdot\left(B^{0}(t, x, v, u)\right)^{-1} \mathcal{B}(t, x, v, u) \mathbb{P} u\right] \mathbb{P}\right|_{\mathrm{op}} \leq|t| \theta+\tilde{\beta}_{2}|\mathbb{P} u|,
\end{gathered}
$$

and

$$
\left|\mathbb{P}\left[D_{u} B^{0}(t, x, v, u) \cdot\left(B^{0}(t, x, v, u)\right)^{-1} \mathcal{B}(t, x, v, u) \mathbb{P} u\right] \mathbb{P}^{\perp}\right|_{\mathrm{op}} \leq|t| \theta+\tilde{\beta}_{3}|\mathbb{P} u|,
$$

hold for all $(t, x, v, u) \in\left[T_{0}, 0\right] \times \mathbb{T}^{n} \times B_{r}\left(\mathbb{R}^{M}\right) \times B_{\mathcal{R}}\left(\mathbb{R}^{N}\right)$, then the bounds (B.5)-(B.6) will be satisfied for all $(t, x, v, u) \in\left[T_{0}, 0\right] \times \mathbb{T}^{n} \times B_{r}\left(\mathbb{R}^{M}\right) \times B_{\mathcal{R}}\left(\mathbb{R}^{N}\right)$ for the constants $\beta_{1}, \beta_{2}, \beta_{3}$ and $\omega$ given by

$$
\beta_{1}=\frac{\tilde{\beta}_{1}\left(\mathcal{R}+\mathcal{R}^{2}\right)}{2}, \quad \beta_{3}=\beta_{2}=\frac{\tilde{\beta}_{2} \sqrt{\left(\mathcal{R}+\mathcal{R}^{2}\right)}}{2} \text { and } \omega=\mathcal{R} .
$$

In particular, this shows that by choosing $\mathcal{R}$ small enough, we can always arrange that $\beta_{1}, \beta_{2}$ and $\beta_{3}$ are as small as we like.

Since the left-hand side of $(\mathbb{B} .6)-(B .8)$ are linear in $\mathbb{P} u$, it is clear that there always exists constants $\tilde{\beta}_{2}, \tilde{\beta}_{3}, \beta_{4}>0$ such that the bounds (B.31)-(B.32) and (B.8) hold for all $(t, x, v, u) \in$ $\left[T_{0}, 0\right] \times \mathbb{T}^{n} \times B_{r}\left(\mathbb{R}^{M}\right) \times B_{\mathcal{R}}\left(\mathbb{R}^{N}\right)$. Moreover, by choosing $\mathcal{R}>0$ small enough, we can take $\beta_{4}>0$ as small as we like.

Thus we can conclude the following:

If there exists a constant $\tilde{\beta}_{1}>0$ such that

$$
\left|\mathbb{P}^{\perp}\left[D_{u} B^{0}(t, x, v, u) \cdot\left(B^{0}(t, x, v, u)\right)^{-1} \mathcal{B}(t, x, v, u) \mathbb{P} u\right] \mathbb{P}^{\perp}\right|_{o p} \leq|t| \theta+\tilde{\beta}_{1}|\mathbb{P} u|^{2}
$$

for all $(t, x, v, u) \in\left[T_{0}, 0\right] \times \mathbb{T}^{n} \times B_{r}\left(\mathbb{R}^{M}\right) \times B_{\mathcal{R}}\left(\mathbb{R}^{N}\right)$ and $\mathcal{R} \in\left(0, \mathcal{R}_{0}\right]$, then there exists constants $\beta_{1}, \beta_{2}, \beta_{3}, \beta_{4}>0$ such that the bounds (B.5) -(B.8) hold for all $(t, x, v, u) \in\left[T_{0}, 0\right] \times \mathbb{T}^{n} \times B_{r}\left(\mathbb{R}^{M}\right) \times$ $B_{\mathcal{R}}\left(\mathbb{R}^{N}\right)$. Moreover, by choosing $\mathcal{R}>0$ small enough, the constants $\beta_{1}, \beta_{2}, \beta_{3}, \beta_{4}$ can be taken arbitrarily small. 


\section{REFERENCES}

1. R.A. Adams and J. Fournier, Sobolev spaces, $2^{\text {nd }}$ ed., Academic Press, 2003.

2. Y. Choquet-Bruhat, General relativity and the Einstein equations, Oxford University Press, 2009.

3. A. Friedman, Partial differential equations, Krieger Publishing Company, 1976.

4. H. Friedrich, On the hyperbolicity of Einstein's and other gauge field equations, Commun. Math. Phys. 100 (1985), $525-543$.

5. _ On the existence of n-geodesically complete or future complete solutions of Einsteins field equations with smooth asymptotic structure, Commun. Math. Phys. 107 (1986), 587-609.

6. - On the global existence and the asymptotic behavior of solutions to the Einstein-Maxwell-Yang-Mills equations, J. Differential Geom. 34 (1991), 275-345.

7. M. Hadžić and J. Speck, The global future stability of the FLRW solutions to the Dust-Einstein system with a positive cosmological constant, J. Hyper. Differential Equations 12 (2015), 87-188.

8. C. Lübbe and J. A. Valiente Kroon, A conformal approach for the analysis of the non-linear stability of radiation cosmologies, Annals of Physics 328 (2013), 1-25.

9. T. A. Oliynyk, Cosmological post-Newtonian expansions to arbitrary order, Commun. Math. Phys. 295 (2010), 431-463.

10. - The cosmological Newtonian limit on cosmological scales, Commun. Math. Phys. 339 (2015), 455-512.

11. H. Ringstöm, Future stability of the Einstein-non-linear scalar field system, Invent. Math. 173 (2008), 123-208.

12. I. Rodnianski and J. Speck, The stability of the irrotational Euler-Einstein system with a positive cosmological constant, J. Eur. Math. Soc. 15 (2013), 2369-2462.

13. J. Speck, The nonlinear future-stability of the FLRW family of solutions to the Euler-Einstein system with a positive cosmological constant, Selecta Mathematica 18 (2012), 633-715.

14. M.E. Taylor, Partial differential equations III: nonlinear equations, Springer, 1996.

School of Mathematical Sciences, Monash University, ViC 3800, Australia

E-mail address: todd.oliynyk@monash.edu 